Article

\title{
On Classification of Symmetry Reductions for the Eikonal Equation
}

\author{
Vasyl Fedorchuk ${ }^{1,2, *}$ and Volodymyr Fedorchuk ${ }^{2}$ \\ 1 Institute of Mathematics, Pedagogical University, 2 Podchorążych St., 30-084 Cracow, Poland \\ 2 Pidstryhach Institute for Applied Problems of Mechanics and Mathematics, Ukr. Nat. Acad. Sci., \\ 3b Naukova St., 79601 Lviv, Ukraine; vasfed@gmail.com \\ * Correspondence: fedorchuk@up.krakow.pl \\ Academic Editor: Antonio Palacios \\ Received: 11 April 2016; Accepted: 7 June 2016; Published: 17 June 2016
}

\begin{abstract}
We study the relationship between the classification of three-dimensional nonconjugate subalgebras of the Lie algebra of the Poincare group $P(1,4)$ and the types of symmetry reduction of the eikonal equation to ordinary differential equations (ODEs).
\end{abstract}

Keywords: symmetry reduction; invariant solutions; eikonal equation; classification of Lie algebras; nonconjugate subalgebras; Poincaré group $P(1,4)$

\section{Introduction}

The eikonal equations in spaces of different dimensions and different types have many applications in the geometric optics, acoustics of inhomogeneous media, theoretical physics, etc. The details on this theme can be found in [1-7] (see also the references therein).

Sheng, Guha and Gonzalez (see [8,9] and the references therein) proposed new effective methods for solving many problems of computational optics.

The complete group classification of the eikonal equations for a two- and three-dimensional nonhomogeneous medium is carried out in [1-4]. In those papers the exact solutions for equations under investigation are also obtained.

In [5], a group classification of generalized eikonal equations was performed. The paper contains a list of all non-equivalent (with respect to equivalence group) equations with symmetry extensions of the kernel. New nonlinear equations of first-order with wide symmetry groups are found.

In [6], the method of propagating waves in multidimensional media is studied in order to find cases of integrability in an explicit form of the eikonal equation.

Many important results conserning symmetry, reduction, and exact solutions of the eikonal equations can be found in the monograph of Fushchych, Shtelen, and Serov [10].

In this paper, we consider the eikonal equation of the form as follows:

$$
\left(\frac{\partial u}{\partial x_{0}}\right)^{2}-\left(\frac{\partial u}{\partial x_{1}}\right)^{2}-\left(\frac{\partial u}{\partial x_{2}}\right)^{2}-\left(\frac{\partial u}{\partial x_{3}}\right)^{2}=1
$$

where $u=u(x), x=\left(x_{0}, x_{1}, x_{2}, x_{3}\right) \in M(1,3)$.

In 1982, Fushchych and Shtelen [11] proved that the maximally extensive local (in sense of Lie) invariance group of Equation (1) was the conformal group $C(1,4)$ of the $(4+1)$-dimensional Poincaré-Minkowski space with the metric

$$
s^{2}=x_{0}^{2}-x_{1}^{2}-x_{2}^{2}-x_{3}^{2}-u^{2}, x_{4}=u
$$


Some exact multiparametrical solutions of Equation (1) are obtained. A procedure of generating new exact solutions from the known ones is also presented.

In [12], using subalgebras of rank 3 of the Lie algebra of the group $C(1,4)$, the ansatzes, which reduce the eikonal equation to ODEs, were constructed. Taking into account the solutions of the reduced equations, wide classes of exact solutions of the eikonal equation were found.

The Lie algebra of the group $C(1,4)$ contains, as subalgebras, the Lie algebras of the following groups: Poincaré group $P(1,4)$, extended Poincaré group $\widetilde{P}(1,4)$, and the optical group Opt $(3)[13,14]$.

In [15], the subalgebras of rank 3 of the Lie algebra of the group Opt(3), which are nonequivalent to subalgebras of the Lie algebra of the group $\widetilde{P}(1,4)$, were used for symmetry reduction of the Equation (1) to ODEs.

In [13], the symmetry reduction was made and some exact solutions for the eikonal equation were constructed using subalgebras of the Lie algebra of the group $\widetilde{P}(1,4)$.

In the papers [16-22], we studied the eikonal equation using the subgroup structure of the proper orthochronous group $P(1,4)$. By using nonconjugate subalgebras of ranks 1,2 , and 3 , we performed the symmetry reduction of the Equation (1) to differential equations with less a number of independent variables. Taking into account the solutions of the reduced equations, some invariant solutions of the eikonal equation were constructed.

However, it turned out that the reduced equations, obtained with the help of nonconjugate subalgebras of the Lie algebra of the group $P(1,4)$ of the given rank, were of different types. Let us present some examples.

It is known that if we reduce nonlinear partial differential equations (PDEs) we obtain, as a rule, nonlinear reduced equations. But, in the cases of the eikonal equation, Euler-Lagrange-Born-Infeld equation, and Monge-Ampere equation in the space $M(1,3) \times R(u)$ we obtained, in some cases, linear ODEs instead of the nonlinear ODEs $[16,21,22]$. Here, and in what follows, $R(u)$ is a real number axis of the dependent variable $\mathrm{u}$.

It is also known (see, for example $[23,24]$ ) that if we reduce PDEs using the nonconjugate subalgebras with a given rank of the Lie algebras of their symmetry groups, we obtain, as a rule, reduced equations with the same number of independent variables. However, in the case of symmetry reduction of the nonlinear wave equation in the space $M(1,4) \times R(u)$ we obtained, in some cases, functional equations, ODEs, two- and three-dimensional PDEs instead of ODEs, two-, three- and four-dimensional reduced PDEs, correspondingly [16,25].

Grundland, Harnad, and Winternitz [26] were the first to point out and investigate the similar phenomenon.

Recently, Nikitin and Kuriksha $[27,28]$ used the three-dimensional nonconjugate subalgebras of the Lie algebra of the Poincare group $P(1,3)$ for symmetry reductions of equations of axion electrodynamics. They obtained four types of reductions. The results obtained cannot be explained in the usual approach (using only the ranks or dimensions of the considered subalgebras).

It means that using only the rank of nonconjugate subalgebras of the Lie algebras of the symmetry groups of the above mentioned equations under investigation, we cannot explain differences in the properties of their reduced equations.

It is known that the nonconjugate subalgebras of the Lie algebra of the group $P(1,4)$ of the same rank may have different structural properties. Therefore, in order to try to explain some of the differences in the properties of the above mentioned reduced equations, we suggest to try to investigate the relationship between structural properties of nonconjugate subalgebras of the same rank of the Lie algebra of the group $P(1,4)$ and the properties of the reduced equations corresponding to them.

In the present paper, we plan to present some of the results concerning the relationship between the structural properties of three-dimensional nonconjugate subalgebras of the Lie algebra of the group $P(1,4)$ and the types of symmetry reduction of the eikonal equation to ODEs. 


\section{The Lie Algebra of the Group $P(1,4)$ and Its Nonconjugate Subalgebras}

The group $P(1,4)$ is a group of rotations and translations of the five-dimensional Minkowski space $M(1,4)$. This group has many applications in the theoretical and mathematical physics (see, for example, $[29,30])$.

The Lie algebra of the group $P(1,4)$ is given by the 15 basis elements $M_{\mu v}=-M_{\nu \mu}$ $(\mu, v=0,1,2,3,4)$ and $P_{\mu}(\mu=0,1,2,3,4)$, satisfying the commutation relations

$$
\begin{gathered}
{\left[P_{\mu}, P_{v}\right]=0, \quad\left[M_{\mu v}, P_{\sigma}\right]=g_{v \sigma} P_{\mu}-g_{\mu \sigma} P_{v}} \\
{\left[M_{\mu v}, M_{\rho \sigma}\right]=g_{\mu \sigma} M_{v \rho}+g_{v \rho} M_{\mu \sigma}-g_{\mu \rho} M_{v \sigma}-g_{v \sigma} M_{\mu \rho}}
\end{gathered}
$$

where $g_{00}=-g_{11}=-g_{22}=-g_{33}=-g_{44}=1, g_{\mu \nu}=0$, if $\mu \neq v$.

In the following, we will use new basis elements

$$
\begin{gathered}
G=M_{04}, \quad L_{1}=M_{23}, \quad L_{2}=-M_{13}, \quad L_{3}=M_{12} \\
P_{a}=M_{a 4}-M_{0 a}, \quad C_{a}=M_{a 4}+M_{0 a}, \quad(a=1,2,3) \\
X_{0}=\frac{1}{2}\left(P_{0}-P_{4}\right), \quad X_{k}=P_{k} \quad(k=1,2,3), \quad X_{4}=\frac{1}{2}\left(P_{0}+P_{4}\right)
\end{gathered}
$$

Let us consider the following representation for the Lie algebra of the group $P(1,4)$ :

$$
\begin{gathered}
P_{0}=\frac{\partial}{\partial x_{0}}, \quad P_{1}=-\frac{\partial}{\partial x_{1}}, \quad P_{2}=-\frac{\partial}{\partial x_{2}}, \quad P_{3}=-\frac{\partial}{\partial x_{3}} \\
P_{4}=-\frac{\partial}{\partial u}, \quad M_{\mu v}=x_{\mu} P_{v}-x_{v} P_{\mu}, x_{4} \equiv u
\end{gathered}
$$

It means that the group $P(1,4)$ acts on the space $M(1,3) \times R(u)$. More details about this type of representations can be found in $[10,31,32]$.

In order to describe nonconjugate subalgebras of the Lie algebra of the group $P(1,4)$, we used a method proposed by Patera, Winternitz, and Zassenhaus in [33].

The subgroup structure of the group $P(1,4)$ was studied in [34-38]. One of the nontrivial consequences of the description of the non-conjugate subalgebras of the Lie algebra of the group $P(1,4)$ is that the Lie algebra of the group $P(1,4)$ contains, as subalgebras, the Lie algebra of the Poincare group $P(1,3)$ and the Lie algebra of the extended Galilei group $\widetilde{G}(1,3)$ [39], i.e., it naturally unites the Lie algebras of the symmetry groups of relativistic and non-relativistic physics.

In the present work, we use the complete list of nonconjugate (up to $P(1,4)$-conjugation) subalgebras of the Lie algebra of the group $P(1,4)$ as given by Fushchich, Barannik, and Barannik in [40].

Taking into account the complete classification of real structures of Lie algebras of a dimension less or equal four obtained by Mubarakzyanov in [41], we classify all the three-dimensional nonconjugate subalgebras of the Lie algebra of the group $P(1,4)$ into classes of isomorphic subalgebras (see, [42]).

\section{Results}

In this section, we present the results obtained while studying the relationship between the structural properties of the three-dimensional nonconjugate subalgebras of the Lie algebra of the group $P(1,4)$ and those obtained through the symmetry reduction of the eikonal equation to ODEs.

In the paper, the symbol $A_{r, j}^{a}$ denotes the jth Lie algebra of dimension $\mathrm{r}$ and $a$ is a continuous parameter for the algebra. It should be indicate that the notation $A_{r, j}^{a}$ corresponds to those used in the paper by Patera et al. [43]. In what follows, for the given specific Lie algebra, we write only nonzero commutation relations $[41,43]$. 


\subsection{Lie Algebras of the Type $3 A_{1}$}

By $3 A_{1}$ we denote the real Lie algebra of dimension three [44]. The Lie algebras of the type $3 A_{1}$ are Abelian.

The Lie algebra of the group $P(1,4)$ contains 31 nonconjugate subalgebras of the type $3 A_{1}$. Below, we present the results obtained for those subalgebras.

\subsubsection{Reductions to Algebraic Equations}

The invariants of five subalgebras allow us to construct the ansatzes, which reduce the eikonal equation to algebraic equations.

(1) $\left\langle P_{1}-\gamma X_{3}, \gamma>0\right\rangle \oplus\left\langle P_{2}-X_{2}-\delta X_{3}, \delta \neq 0\right\rangle \oplus\left\langle X_{4}\right\rangle$.

Ansatz:

$$
x_{3}\left(x_{0}+u\right)^{2}-\left(\gamma x_{1}+x_{2} \delta-x_{3}\right)\left(x_{0}+u\right)-\gamma x_{1}=\varphi(\omega), \quad \omega=x_{0}+u
$$

Reduced equation:

$$
\omega^{4}+2 \omega^{3}+\left(\gamma^{2}+\delta^{2}+1\right) \omega^{2}+2 \gamma^{2} \omega+\gamma^{2}=0
$$

Solution of the eikonal equation:

$$
\left(x_{0}+u\right)^{4}+2\left(x_{0}+u\right)^{3}+\left(\gamma^{2}+\delta^{2}+1\right)\left(x_{0}+u\right)^{2}+2 \gamma^{2}\left(x_{0}+u\right)+\gamma^{2}=0
$$

(2) $\left\langle P_{1}-\gamma X_{3}, \gamma>0\right\rangle \oplus\left\langle P_{2}-X_{2}\right\rangle \oplus\left\langle X_{4}\right\rangle$.

Ansatz:

$$
x_{3}\left(x_{0}+u\right)^{2}-\left(\gamma x_{1}-x_{3}\right)\left(x_{0}+u\right)-\gamma x_{1}=\varphi(\omega), \quad \omega=x_{0}+u
$$

Reduced equation:

$$
(\omega+1)^{2}\left(\omega^{2}+\gamma^{2}\right)=0
$$

Solutions of the eikonal equation:

$$
u=-1-x_{0},\left(x_{0}+u\right)^{2}+\gamma^{2}=0
$$

(3) $\left\langle P_{1}\right\rangle \oplus\left\langle P_{2}-X_{2}-\delta X_{3}, \delta>0\right\rangle \oplus\left\langle X_{4}\right\rangle$.

Ansatz:

$$
x_{3}\left(x_{0}+u\right)-x_{2} \delta+x_{3}=\varphi(\omega), \quad \omega=x_{0}+u
$$

Reduced equation:

$$
\omega^{2}+2 \omega+\delta^{2}-1=0
$$

Solution of the eikonal equation:

$$
\left(x_{0}+u\right)^{2}+2\left(x_{0}+u\right)+\delta^{2}+1=0
$$

As we see, the left hand sides of the Ansatzes (1)-(3) are polinomials in invariant $\omega=x_{0}+u$. The reduced equations are also polinomials in $\omega$, but with the constant coefficients. The solutions of the eikonal equation are also polinomials in variable $x_{0}+u$ with the constant coefficients.

(4) $\left\langle P_{1}-X_{3}\right\rangle \oplus\left\langle P_{2}\right\rangle \oplus\left\langle X_{4}\right\rangle$.

Ansatz:

$$
x_{3}-\frac{x_{1}}{x_{0}+u}=\varphi(\omega), \quad \omega=x_{0}+u
$$

Reduced equation:

$$
\left(1+\omega^{2}\right) \omega^{2}=0
$$


Solutions of the reduced equation:

$$
1+\omega^{2}=0, \quad \omega=0
$$

Solutions of the eikonal equation:

$$
1+\left(x_{0}+u\right)^{2}=0, u=-x_{0}
$$

(5) $\left\langle P_{3}-X_{2}\right\rangle \oplus\left\langle X_{1}\right\rangle \oplus\left\langle X_{4}\right\rangle$.

Ansatz:

$$
x_{2}-\frac{x_{3}}{x_{0}+u}=\varphi(\omega), \quad \omega=x_{0}+u
$$

Reduced equation:

$$
\left(\omega^{2}+1\right) \omega^{2}=0
$$

Solutions of the reduced equation:

$$
\omega^{2}+1=0, \quad \omega=0
$$

Solutions of the eikonal equation:

$$
\left(x_{0}+u\right)^{2}+1=0, u=-x_{0}
$$

\subsubsection{Reductions to Linear ODEs}

The invariants of six subalgebras allow us to construct the ansatzes, which reduce the eikonal equation to linear ODEs.

(1) $\left\langle P_{1}\right\rangle \oplus\left\langle P_{2}\right\rangle \oplus\left\langle X_{3}\right\rangle$.

Ansatz:

$$
x_{0}^{2}-x_{1}^{2}-x_{2}^{2}-u^{2}=\varphi(\omega), \quad \omega=x_{0}+u
$$

Reduced equation:

$$
\omega \varphi^{\prime}-\varphi=0
$$

Solution of the reduced equation:

$$
\varphi(\omega)=c_{1} \omega
$$

Solution of the eikonal equation:

$$
x_{0}^{2}-x_{1}^{2}-x_{2}^{2}-u^{2}=c_{1}\left(x_{0}+u\right)
$$

(2) $\left\langle P_{3}\right\rangle \oplus\left\langle X_{1}\right\rangle \oplus\left\langle X_{2}\right\rangle$.

Ansatz:

$$
x_{0}^{2}-x_{3}^{2}-u^{2}=\varphi(\omega), \quad \omega=x_{0}+u
$$

Reduced equation:

$$
\omega \varphi^{\prime}-\varphi=0
$$

Solution of the reduced equation:

$$
\varphi(\omega)=c_{1} \omega
$$


Solution of the eikonal equation:

$$
x_{0}^{2}-x_{3}^{2}-u^{2}=c_{1}\left(x_{0}+u\right)
$$

(3) $\left\langle P_{1}\right\rangle \oplus\left\langle P_{2}\right\rangle \oplus\left\langle P_{3}\right\rangle$.

Ansatz:

$$
x_{0}^{2}-x_{1}^{2}-x_{2}^{2}-x_{3}^{2}-u^{2}=\varphi(\omega), \quad \omega=x_{0}+u
$$

Reduced equation:

$$
\omega \varphi^{\prime}-\varphi=0
$$

Solution of the reduced equation:

$$
\varphi(\omega)=c_{1} \omega
$$

Solution of the eikonal equation:

$$
x_{0}^{2}-x_{1}^{2}-x_{2}^{2}-x_{3}^{2}-u^{2}=c_{1}\left(x_{0}+u\right)
$$

Let us note, that in the cases (1)-(3) we obtained the same reduced equations.

(4) $\left\langle P_{1}\right\rangle \oplus\left\langle P_{2}-X_{2}\right\rangle \oplus\left\langle X_{3}\right\rangle$.

Ansatz:

$$
\frac{x_{0}^{2}-x_{1}^{2}-u^{2}}{x_{0}+u}-\frac{x_{2}^{2}}{x_{0}+u+1}=\varphi(\omega), \quad \omega=x_{0}+u
$$

Reduced equation:

$$
\varphi^{\prime}(\omega+1)^{4} \omega^{4}=0
$$

Solutions of the reduced equation:

$$
\varphi(\omega)=c_{1}, \quad \omega+1=0, \quad \omega=0
$$

Solutions of the eikonal equation:

$$
\frac{x_{0}^{2}-x_{1}^{2}-u^{2}}{x_{0}+u}-\frac{x_{2}^{2}}{x_{0}+u+1}=c_{1}, u=-1-x_{0}, \quad u=-x_{0}
$$

(5) $\left\langle P_{1}\right\rangle \oplus\left\langle P_{2}-\alpha X_{2}, \alpha>0\right\rangle \oplus\left\langle P_{3}-\gamma X_{3}, \gamma \neq 0\right\rangle$.

Ansatz:

$$
2 u+\frac{x_{1}^{2}}{x_{0}+u}+\frac{x_{2}^{2}}{x_{0}+u+\alpha}+\frac{x_{3}^{2}}{x_{0}+u+\gamma}=\varphi(\omega), \quad \omega=x_{0}+u
$$

The reduced equation:

$$
\omega^{4}(\omega+\gamma)^{4}(\omega+\alpha)^{4}\left(\varphi^{\prime}-1\right)=0
$$

Solutions of the reduced equation:

$$
\omega=0, \quad \omega+\gamma=0, \quad \omega+\alpha=0, \quad \varphi(\omega)=\omega+c
$$

Solutions on the eikonal equation:

$$
\begin{gathered}
u=-x_{0}, \quad u=-x_{0}-\gamma, \quad u=-x_{0}-\alpha \\
2 u+\frac{x_{1}^{2}}{x_{0}+u}+\frac{x_{2}^{2}}{x_{0}+u+\alpha}+\frac{x_{3}^{2}}{x_{0}+u+\gamma}=x_{0}+u+c
\end{gathered}
$$


(6) $\left\langle P_{1}\right\rangle \oplus\left\langle P_{2}-\alpha X_{2}, \alpha>0\right\rangle \oplus\left\langle P_{3}\right\rangle$.

Ansatz:

$$
2 u+\frac{x_{1}^{2}+x_{3}^{2}}{x_{0}+u}+\frac{x_{2}^{2}}{x_{0}+u+\alpha}=\varphi(\omega), \quad \omega=x_{0}+u
$$

The reduced equation:

$$
\omega^{4}(\omega+\alpha)^{4}\left(\varphi^{\prime}-1\right)=0
$$

Solutions of the reduced equation:

$$
\omega=0, \quad \omega+\alpha=0, \quad \varphi(\omega)=\omega+c
$$

Solutions on the eikonal equation:

$$
u=-x_{0}, \quad u=-x_{0}-\alpha, \quad 2 u+\frac{x_{1}^{2}+x_{3}^{2}}{x_{0}+u}+\frac{x_{2}^{2}}{x_{0}+u+\alpha}=x_{0}+u+c
$$

\subsubsection{Reductions to Equations, Which Can Be Split on Two Linear ODEs}

Taking into account the invariants of nine nonconjugate subalgebras, we constructed the ansatzes, which reduced the eikonal equation to those, which could be split on two linear ODEs.

(1) $\left\langle X_{1}\right\rangle \oplus\left\langle X_{2}\right\rangle \oplus\left\langle X_{4}\right\rangle$.

Ansatz:

$$
x_{0}+u=\varphi(\omega), \quad \omega=x_{3}
$$

Reduced equation:

$$
\left(\varphi^{\prime}\right)^{2}=0
$$

Solution of the reduced equation:

$$
\varphi(\omega)=c_{1}
$$

Solution of the eikonal equation:

$$
u=c_{1}-x_{0}
$$

(2) $\left\langle P_{1}\right\rangle \oplus\left\langle P_{2}-X_{2}\right\rangle \oplus\left\langle X_{4}\right\rangle$.

Ansatz:

$$
x_{0}+u=\varphi(\omega), \quad \omega=x_{3}
$$

Reduced equation:

$$
\left(\varphi^{\prime}\right)^{2}=0
$$

Solution of the reduced equation:

$$
\varphi(\omega)=c_{1}
$$

Solution of the eikonal equation:

$$
u=c_{1}-x_{0}
$$

(3) $\left\langle L_{3}\right\rangle \oplus\left\langle X_{3}\right\rangle \oplus\left\langle X_{4}\right\rangle$.

Ansatz:

$$
x_{0}+u=\varphi(\omega), \quad \omega=\left(x_{1}^{2}+x_{2}^{2}\right)^{1 / 2}
$$

Reduced equation:

$$
\left(\varphi^{\prime}\right)^{2}=0
$$


Solution of the reduced equation:

$$
\varphi(\omega)=c_{1}
$$

Solution of the eikonal equation:

$$
u=c_{1}-x_{0}
$$

(4) $\left\langle P_{3}\right\rangle \oplus\left\langle X_{1}\right\rangle \oplus\left\langle X_{4}\right\rangle$.

Ansatz:

$$
x_{0}+u=\varphi(\omega), \quad \omega=x_{2}
$$

Reduced equation:

$$
\left(\varphi^{\prime}\right)^{2}=0
$$

Solution of the reduced equation:

$$
\varphi(\omega)=c_{1}
$$

Solution of the eikonal equation:

$$
u=c_{1}-x_{0}
$$

(5) $\left\langle L_{3}\right\rangle \oplus\left\langle P_{3}\right\rangle \oplus\left\langle X_{4}\right\rangle$.

Ansatz:

$$
x_{0}+u=\varphi(\omega), \quad \omega=\left(x_{1}^{2}+x_{2}^{2}\right)^{1 / 2}
$$

Reduced equation:

$$
\left(\varphi^{\prime}\right)^{2}=0
$$

Solution of the reduced equation:

$$
\varphi(\omega)=c_{1}
$$

Solution of the eikonal equation:

$$
u=c_{1}-x_{0}
$$

(6) $\left\langle P_{1}\right\rangle \oplus\left\langle P_{2}\right\rangle \oplus\left\langle X_{4}\right\rangle$.

Ansatz:

$$
x_{0}+u=\varphi(\omega), \quad \omega=x_{3}
$$

Reduced equation:

$$
\left(\varphi^{\prime}\right)^{2}=0
$$

Solution of the reduced equation:

$$
\varphi(\omega)=c_{1}
$$

Solution of the eikonal equation:

$$
u=c_{1}-x_{0}
$$

Let us note that, in the cases (1)-(6), we obtained the same reduced equations. The solutions of the eikonal equation are also the same.

(7) $\langle G\rangle \oplus\left\langle X_{2}\right\rangle \oplus\left\langle X_{1}\right\rangle$.

Ansatz:

$$
\left(x_{0}^{2}-u^{2}\right)^{1 / 2}=\varphi(\omega), \quad \omega=x_{3}
$$

Reduced equation:

$$
\left(\varphi^{\prime}-1\right)\left(\varphi^{\prime}+1\right)=0
$$


Solutions of the reduced equation:

$$
\varphi(\omega)=\varepsilon \omega+c_{1}, \varepsilon= \pm 1
$$

Solutions of the eikonal equation:

$$
\left(x_{0}^{2}-u^{2}\right)^{1 / 2}=\varepsilon x_{3}+c_{1}, \varepsilon= \pm 1
$$

(8) $\langle G\rangle \oplus\left\langle L_{3}\right\rangle \oplus\left\langle X_{3}\right\rangle$.

Ansatz:

$$
\left(x_{0}^{2}-u^{2}\right)^{1 / 2}=\varphi(\omega), \quad \omega=\left(x_{1}^{2}+x_{2}^{2}\right)^{1 / 2}
$$

Reduced equation:

$$
\left(\varphi^{\prime}-1\right)\left(\varphi^{\prime}+1\right)=0
$$

Solutions of the reduced equation:

$$
\varphi(\omega)=\varepsilon \omega+c_{1}, \varepsilon= \pm 1
$$

Solutions of the eikonal equation:

$$
\left(x_{0}^{2}-u^{2}\right)^{1 / 2}=\varepsilon\left(x_{1}^{2}+x_{2}^{2}\right)^{1 / 2}+c_{1}, \varepsilon= \pm 1
$$

(9) $\left\langle P_{3}-2 X_{0}\right\rangle \oplus\left\langle X_{1}\right\rangle \oplus\left\langle X_{2}\right\rangle$.

Ansatz:

$$
\frac{1}{6}\left(x_{0}+u\right)^{3}+x_{3}\left(x_{0}+u\right)+x_{0}-u=\varphi(\omega), \quad \omega=\left(x_{0}+u\right)^{2}+4 x_{3}
$$

Reduced equation:

$$
16\left(\varphi^{\prime}\right)^{2}-\omega=0
$$

Solutions of the reduced equation:

$$
\varphi(\omega)=\frac{\varepsilon}{6} \omega^{3 / 2}+c_{1}, \varepsilon= \pm 1
$$

Solutions of the eikonal equation:

$$
\frac{1}{6}\left(x_{0}+u\right)^{3}+x_{3}\left(x_{0}+u\right)+x_{0}-u=\frac{\varepsilon}{6}\left(\left(x_{0}+u\right)^{2}+4 x_{3}\right)^{3 / 2}+c_{1}, \varepsilon= \pm 1
$$

\subsubsection{Reductions to Nonlinear ODEs}

From the invariants of five nonconjugate subalgebras we constructed the ansatzes, which reduced the eikonal equation to nonlinear ODEs.

(1) $\left\langle G+\alpha X_{3}, \alpha>0\right\rangle \oplus\left\langle X_{1}\right\rangle \oplus\left\langle X_{2}\right\rangle$.

Ansatz:

$$
x_{3}-\alpha \ln \left(x_{0}+u\right)=\varphi(\omega), \quad \omega=x_{0}^{2}-u^{2}
$$

Reduced equation:

$$
4 \omega\left(\varphi^{\prime}\right)^{2}+4 \alpha \varphi^{\prime}-1=0
$$


Solutions of the reduced equation:

$$
\varphi(\omega)=\varepsilon\left(\alpha^{2}+\omega\right)^{1 / 2}-\varepsilon \alpha \operatorname{artanh} \frac{\left(\alpha^{2}+\omega\right)^{1 / 2}}{\alpha}-\frac{\alpha}{2} \ln (\omega)+c_{1}, \varepsilon= \pm 1
$$

Solutions of the eikonal equation:

$$
x_{3}-\alpha \ln \left(x_{0}+u\right)=\varepsilon\left(\alpha^{2}+x_{0}^{2}-u^{2}\right)^{1 / 2}-\varepsilon \alpha \operatorname{artanh} \frac{\left(\alpha^{2}+x_{0}^{2}-u^{2}\right)^{1 / 2}}{\alpha}-\frac{\alpha}{2} \ln \left(x_{0}^{2}-u^{2}\right)+c_{1}, \varepsilon= \pm 1
$$

(2) $\left\langle L_{3}\right\rangle \oplus\left\langle P_{3}+C_{3}\right\rangle \oplus\left\langle X_{0}+X_{4}\right\rangle$.

Ansatz:

$$
\left(x_{3}^{2}+u^{2}\right)^{1 / 2}=\varphi(\omega), \quad \omega=\left(x_{1}^{2}+x_{2}^{2}\right)^{1 / 2}
$$

Reduced equation:

$$
\left(1+\left(\varphi^{\prime}\right)^{2}\right) \varphi^{2}=0
$$

Solutions of the reduced equation:

$$
\varphi(\omega)=i \varepsilon \omega+c_{1}, \varepsilon= \pm 1 ; \varphi=0
$$

Solutions of the eikonal equation:

$$
\left(x_{3}^{2}+u^{2}\right)^{1 / 2}=i \varepsilon\left(x_{1}^{2}+x_{2}^{2}\right)^{1 / 2}+c_{1}, \varepsilon= \pm 1 ; x_{3}^{2}+u^{2}=0
$$

(3) $\left\langle L_{3}+\alpha\left(X_{0}+X_{4}\right), \alpha>0\right\rangle \oplus\left\langle X_{3}\right\rangle \oplus\left\langle X_{4}\right\rangle$.

Ansatz:

$$
x_{0}+u+\alpha \arctan \frac{x_{2}}{x_{1}}=\varphi(\omega), \quad \omega=\left(x_{1}^{2}+x_{2}^{2}\right)^{1 / 2}
$$

Reduced equation:

$$
\omega^{2}\left(\varphi^{\prime}\right)^{2}+\alpha^{2}=0
$$

Solutions of the reduced equation:

$$
\varphi(\omega)=i \varepsilon \alpha \ln (\omega)+c_{1}, \varepsilon= \pm 1
$$

Solutions of the eikonal equation:

$$
x_{0}+u+\alpha \arctan \frac{x_{2}}{x_{1}}=i \frac{\varepsilon \alpha}{2} \ln \left(x_{1}^{2}+x_{2}^{2}\right)+c_{1}, \varepsilon= \pm 1
$$

(4) $\left\langle P_{3}-2 X_{0}\right\rangle \oplus\left\langle X_{1}\right\rangle \oplus\left\langle X_{4}\right\rangle$.

Ansatz:

$$
\left(x_{0}+u\right)^{2}+4 x_{3}=\varphi(\omega), \quad \omega=x_{2}
$$

Reduced equation:

$$
\left(\varphi^{\prime}\right)^{2}+16=0
$$

Solutions of the reduced equation:

$$
\varphi(\omega)=4 i \varepsilon \omega+c_{1}, \varepsilon= \pm 1
$$

Solutions of the eikonal equation:

$$
\left(x_{0}+u\right)^{2}+4 x_{3}=4 i \varepsilon x_{2}+c_{1}, \varepsilon= \pm 1
$$


(5) $\left\langle L_{3}\right\rangle \oplus\left\langle-P_{3}+2 X_{0}\right\rangle \oplus\left\langle 2 X_{4}\right\rangle$.

Ansatz:

$$
\left(x_{0}+u\right)^{2}+4 x_{3}=\varphi(\omega), \quad \omega=\left(x_{1}^{2}+x_{2}^{2}\right)^{1 / 2}
$$

Reduced equation:

$$
\left(\varphi^{\prime}\right)^{2}+16=0
$$

Solutions of the reduced equation:

$$
\varphi(\omega)=4 i \varepsilon \omega+c_{1}, \varepsilon= \pm 1
$$

Solutions of the eikonal equation:

$$
\left(x_{0}+u\right)^{2}+4 x_{3}=4 i \varepsilon\left(x_{1}^{2}+x_{2}^{2}\right)^{1 / 2}+c_{1}, \varepsilon= \pm 1
$$

\subsubsection{There Are No Reductions}

From the invariants of remaining six nonconjugate subalgebras, it is impossible to construct the ansatzes, which reduce the eikonal equation.

Below, we present those subalgebras as well as their invariants.

(1) $\left\langle L_{3}\right\rangle \oplus\left\langle X_{4}-X_{0}\right\rangle \oplus\left\langle X_{3}\right\rangle: x_{0},\left(x_{1}^{2}+x_{2}^{2}\right)^{1 / 2}$;

(2) $\left\langle L_{3}\right\rangle \oplus\left\langle X_{0}+X_{4}\right\rangle \oplus\left\langle X_{4}-X_{0}\right\rangle: x_{3},\left(x_{1}^{2}+x_{2}^{2}\right)^{1 / 2}$;

(3) $\left\langle X_{1}\right\rangle \oplus\left\langle X_{2}\right\rangle \oplus\left\langle X_{4}-X_{0}\right\rangle: x_{0}, x_{3}$;

(4) $\left\langle X_{0}\right\rangle \oplus\left\langle X_{1}\right\rangle \oplus\left\langle X_{4}\right\rangle: x_{2}, x_{3}$;

(5) $\left\langle L_{3}+\alpha\left(X_{0}+X_{4}\right), \alpha>0\right\rangle \oplus\left\langle X_{3}\right\rangle \oplus\left\langle X_{4}-X_{0}\right\rangle:\left(x_{1}^{2}+x_{2}^{2}\right)^{1 / 2}, x_{0}+\alpha \arctan \frac{x_{2}}{x_{1}}$;

(6) $\left\langle L_{3}+\alpha X_{3}, \alpha>0\right\rangle \oplus\left\langle X_{0}+X_{4}\right\rangle \oplus\left\langle X_{4}-X_{0}\right\rangle:\left(x_{1}^{2}+x_{2}^{2}\right)^{1 / 2}, x_{3}+\alpha \arctan \frac{x_{1}}{x_{2}}$.

3.2. Lie Algebras of the Type $A_{2} \oplus A_{1}$

$$
\left[e_{1}, e_{2}\right]=e_{2}
$$

By $A_{1}$ we denote the real Lie algebra of dimension one [44]. It is known that there are only two different types of the real two-dimensional Lie algebras: decomposable $A_{1} \oplus A_{1} \equiv 2 A_{1}$ and indecomposable $A_{2}$ [41]. The Lie algebras of the type $2 A_{1}$ are Abelian. Bases elements $\left(e_{1}\right.$ and $\left.e_{2}\right)$ of the Lie algebras of type $A_{2}$ satisfy the commutation relations: $\left[e_{1}, e_{2}\right]=e_{2}$ [43]. The Lie algebras of the type $A_{2}$ are solvable $([41,43])$.

The Lie algebra of the group $P(1,4)$ contains 10 nonconjugate subalgebras of the Type $A_{2} \oplus A_{1}$. Below, we present the results obtained for those subalgebras.

\subsubsection{Reductions to Equations, Which Can Be Split on Two Linear ODEs}

Taking into account the invariants of two nonconjugate subalgebras, we constructed the ansatzes, which reduced the eikonal equation to those, which could be split on two linear ODEs.

(1) $\left\langle-G, P_{3}\right\rangle \oplus\left\langle X_{1}\right\rangle$.

Ansatz:

$$
\left(x_{0}^{2}-x_{3}^{2}-u^{2}\right)^{1 / 2}=\varphi(\omega), \quad \omega=x_{2}
$$

Reduced equation:

$$
\left(\varphi^{\prime}-1\right)\left(\varphi^{\prime}+1\right) \varphi^{2}=0
$$


Solutions of the reduced equation:

$$
\varphi(\omega)=\varepsilon \omega+c_{1}, \quad \varphi=0, \quad \varepsilon= \pm 1
$$

Solutions of the eikonal equation:

$$
\left(x_{0}^{2}-x_{3}^{2}-u^{2}\right)^{1 / 2}=\varepsilon x_{2}+c_{1}, \quad x_{0}^{2}-x_{3}^{2}-u^{2}=0, \quad \varepsilon= \pm 1
$$

(2) $\left\langle-G, P_{3}\right\rangle \oplus\left\langle L_{3}\right\rangle$.

Ansatz:

$$
\left(x_{0}^{2}-x_{3}^{2}-u^{2}\right)^{1 / 2}=\varphi(\omega), \quad \omega=\left(x_{1}^{2}+x_{2}^{2}\right)^{1 / 2}
$$

Reduced equation:

$$
\left(\varphi^{\prime}-1\right)\left(\varphi^{\prime}+1\right) \varphi^{2}=0
$$

Solutions of the reduced equation:

$$
\varphi(\omega)=\varepsilon \omega+c_{1}, \quad \varphi=0, \quad \varepsilon= \pm 1
$$

Solutions of the eikonal equation:

$$
\left(x_{0}^{2}-x_{3}^{2}-u^{2}\right)^{1 / 2}=\varepsilon\left(x_{1}^{2}+x_{2}^{2}\right)^{1 / 2}+c_{1}, \quad x_{0}^{2}-x_{3}^{2}-u^{2}=0, \quad \varepsilon= \pm 1
$$

\subsubsection{Reductions to Nonlinear ODEs}

From the invariants of five nonconjugate subalgebras we constructed the ansatzes, which reduced the eikonal equation to non-linear ODEs.

(1) $\left\langle-\left(G+\alpha X_{2}\right), P_{3}, \alpha>0\right\rangle \oplus\left\langle X_{1}\right\rangle$.

Ansatz:

$$
x_{2}-\alpha \ln \left(x_{0}+u\right)=\varphi(\omega), \quad \omega=\left(x_{0}^{2}-x_{3}^{2}-u^{2}\right)^{1 / 2}
$$

Reduced equation:

$$
\omega\left(\omega\left(\varphi^{\prime}\right)^{2}+2 \alpha \varphi^{\prime}-\omega\right)=0
$$

Solutions of the reduced equation:

$$
\omega=0, \varphi(\omega)=\varepsilon\left(\alpha^{2}+\omega^{2}\right)^{1 / 2}-\varepsilon \alpha \operatorname{artanh} \frac{\alpha}{\left(\alpha^{2}+\omega^{2}\right)^{1 / 2}}-\alpha \ln (\omega)+c_{1}, \varepsilon= \pm 1
$$

Solutions of the eikonal equation:

$$
\begin{gathered}
x_{0}^{2}-x_{3}^{2}-u^{2}=0 \\
x_{2}-\alpha \ln \left(x_{0}+u\right)=\varepsilon\left(x_{0}^{2}-x_{3}^{2}-u^{2}+\alpha^{2}\right)^{1 / 2} \\
-\varepsilon \alpha \operatorname{artanh} \frac{\alpha}{\left(x_{0}^{2}-x_{3}^{2}-u^{2}+\alpha^{2}\right)^{1 / 2}} \\
-\frac{\alpha}{2} \ln \left(x_{0}^{2}-x_{3}^{2}-u^{2}\right)+c_{1} \\
\varepsilon= \pm 1
\end{gathered}
$$

(2) $\left\langle-\frac{1}{\lambda} L_{3}-G, 2 X_{4}, \lambda>0\right\rangle \oplus\left\langle X_{3}\right\rangle$. 
Ansatz:

$$
\ln \left(x_{0}+u\right)+\lambda \arctan \frac{x_{1}}{x_{2}}=\varphi(\omega), \quad \omega=\left(x_{1}^{2}+x_{2}^{2}\right)^{1 / 2}
$$

Reduced equation:

$$
\omega^{2}\left(\varphi^{\prime}\right)^{2}+\lambda^{2}=0
$$

Solutions of the reduced equation:

$$
\varphi(\omega)=i \varepsilon \lambda \ln (\omega)+c_{1}, \quad \varepsilon= \pm 1
$$

Solutions of the eikonal equation:

$$
\ln \left(x_{0}+u\right)+\lambda \arctan \frac{x_{1}}{x_{2}}=i \frac{\varepsilon \lambda}{2} \ln \left(x_{1}^{2}+x_{2}^{2}\right)+c_{1}, \quad \varepsilon= \pm 1
$$

(3) $\left\langle-\left(G+\alpha X_{2}\right), X_{4}, \alpha>0\right\rangle \oplus\left\langle X_{1}\right\rangle$.

Ansatz:

$$
x_{2}-\alpha \ln \left(x_{0}+u\right)=\varphi(\omega), \quad \omega=x_{3}
$$

Reduced equation:

$$
\left(\varphi^{\prime}\right)^{2}+1=0
$$

Solutions of the reduced equation:

$$
\varphi(\omega)=i \varepsilon \omega+c_{1}, \quad \varepsilon= \pm 1
$$

Solutions of the eikonal equation:

$$
x_{2}-\alpha \ln \left(x_{0}+u\right)=i \varepsilon x_{3}+c_{1}, \quad \varepsilon= \pm 1
$$

(4) $\left\langle-\left(G+\alpha X_{3}\right), X_{4}, \alpha>0\right\rangle \oplus\left\langle L_{3}+\beta X_{3}, \beta>0\right\rangle$.

Ansatz:

$$
x_{3}-\alpha \ln \left(x_{0}+u\right)+\beta \arctan \frac{x_{1}}{x_{2}}=\varphi(\omega), \quad \omega=\left(x_{1}^{2}+x_{2}^{2}\right)^{1 / 2}
$$

Reduced equation:

$$
\omega^{2}\left(\varphi^{\prime}\right)^{2}+\omega^{2}+\beta^{2}=0
$$

Solutions of the reduced equation:

$$
\varphi(\omega)=i \varepsilon\left(\omega^{2}+\beta^{2}\right)^{1 / 2}-i \varepsilon \beta \operatorname{artanh} \frac{\beta}{\left(\omega^{2}+\beta^{2}\right)^{1 / 2}}+c_{1}, \quad \varepsilon= \pm 1
$$

Solutions of the eikonal equation:

$$
\begin{gathered}
x_{3}-\alpha \ln \left(x_{0}+u\right)+\beta \arctan \frac{x_{1}}{x_{2}}=-i \varepsilon \beta \operatorname{artanh} \frac{\beta}{\left(x_{1}^{2}+x_{2}^{2}+\beta^{2}\right)^{1 / 2}}+i \varepsilon\left(x_{1}^{2}+x_{2}^{2}+\beta^{2}\right)^{1 / 2}+c_{1} \\
\varepsilon= \pm 1
\end{gathered}
$$

(5) $\left\langle-\left(G+\alpha X_{3}\right), X_{4}, \alpha>0\right\rangle \oplus\left\langle L_{3}\right\rangle$.

Ansatz:

$$
x_{3}-\alpha \ln \left(x_{0}+u\right)=\varphi(\omega), \quad \omega=\left(x_{1}^{2}+x_{2}^{2}\right)^{1 / 2}
$$


Reduced equation:

$$
\left(\varphi^{\prime}\right)^{2}+1=0
$$

Solutions of the reduced equation:

$$
\varphi(\omega)=i \varepsilon \omega+c_{1}, \quad \varepsilon= \pm 1
$$

Solutions of the eikonal equation:

$$
x_{3}-\alpha \ln \left(x_{0}+u\right)=i \varepsilon\left(x_{1}^{2}+x_{2}^{2}\right)^{1 / 2}+c_{1}, \quad \varepsilon= \pm 1
$$

\subsubsection{There Are No Reductions}

From the invariants of remaining three nonconjugate subalgebras, it is impossible to construct the ansatzes, which reduce the eikonal equation.

Below, we present those subalgebras as well as their invariants.

(1) $\left\langle-G, X_{4}\right\rangle \oplus\left\langle X_{1}\right\rangle: x_{2}, x_{3}$;

(2) $\left\langle-G, X_{4}\right\rangle \oplus\left\langle L_{3}\right\rangle: x_{3},\left(x_{1}^{2}+x_{2}^{2}\right)^{1 / 2}$;

(3) $\left\langle-G, X_{4}\right\rangle \oplus\left\langle L_{3}+\alpha X_{3}, \alpha>0\right\rangle:\left(x_{1}^{2}+x_{2}^{2}\right)^{1 / 2}, x_{3}+\alpha \arctan \frac{x_{1}}{x_{2}}$.

3.3. Lie Algebras of the Type $A_{3,1}$

$$
\left[e_{2}, e_{3}\right]=e_{1}
$$

Lie algebras of the type $A_{3,1}$ are nilpotent [43].

The Lie algebra of the group $P(1,4)$ contains 17 nonconjugate subalgebras of the type $A_{3,1}$. Below, we present the results obtained for those subalgebras.

\subsubsection{Reductions to Algebraic Equations}

Taking into account the invariants of seven nonconjugate subalgebras, we constructed the ansatzes, which reduced the eikonal equation to algebraic equations.

(1) $\left\langle 4 X_{4}, P_{1}-X_{2}-\gamma X_{3}, P_{2}+X_{1}-\mu X_{2}-\delta X_{3}, \gamma>0, \delta \neq 0, \mu>0\right\rangle$.

Ansatz:

$$
x_{3}\left(x_{0}+u\right)^{2}-\left(\gamma x_{1}+x_{2} \delta-\mu x_{3}\right)\left(x_{0}+u\right)+(\delta-\gamma \mu) x_{1}-x_{2} \gamma+x_{3}=\varphi(\omega), \omega=x_{0}+u
$$

Reduced equation:

$$
\omega^{4}+2 \mu \omega^{3}+\left(\gamma^{2}+\mu^{2}+\delta^{2}+2\right) \omega^{2}+2 \mu\left(\gamma^{2}+1\right) \omega+(\gamma \mu-\delta)^{2}+\gamma^{2}+1=0
$$

Solution of the eikonal equation:

$$
\left(x_{0}+u\right)^{4}+2 \mu\left(x_{0}+u\right)^{3}+\left(\gamma^{2}+\mu^{2}+\delta^{2}+2\right)\left(x_{0}+u\right)^{2}+2 \mu\left(\gamma^{2}+1\right)\left(x_{0}+u\right)+(\gamma \mu-\delta)^{2}+\gamma^{2}+1=0
$$

(2) $\left\langle 4 X_{4}, P_{1}-X_{2}-\gamma X_{3}, P_{2}+X_{1}-\mu X_{2}, \gamma>0, \mu>0\right\rangle$.

Ansatz:

$$
x_{3}\left(x_{0}+u\right)^{2}-\left(\gamma x_{1}-\mu x_{3}\right)\left(x_{0}+u\right)-\gamma \mu x_{1}-x_{2} \gamma+x_{3}=\varphi(\omega), \quad \omega=x_{0}+u
$$


Reduced equation:

$$
\omega^{4}+2 \mu \omega^{3}+\left(\gamma^{2}+\mu^{2}+2\right) \omega^{2}+2 \mu\left(\gamma^{2}+1\right) \omega+\gamma^{2}\left(\mu^{2}+1\right)+1=0
$$

Solution of the eikonal equation:

$$
\left(x_{0}+u\right)^{4}+2 \mu\left(x_{0}+u\right)^{3}+\left(\gamma^{2}+\mu^{2}+2\right)\left(x_{0}+u\right)^{2}+2 \mu\left(\gamma^{2}+1\right)\left(x_{0}+u\right)+\gamma^{2}\left(\mu^{2}+1\right)+1=0
$$

(3) $\left\langle 4 X_{4}, P_{1}-X_{2}, P_{2}+X_{1}-\mu X_{2}-\delta X_{3}, \delta>0, \mu \neq 0\right\rangle$.

Ansatz:

$$
x_{3}\left(x_{0}+u\right)^{2}-\left(x_{2} \delta-\mu x_{3}\right)\left(x_{0}+u\right)+\delta x_{1}+x_{3}=\varphi(\omega), \quad \omega=x_{0}+u
$$

Reduced equation:

$$
\omega^{4}+2 \mu \omega^{3}+\left(\delta^{2}+\mu^{2}+2\right) \omega^{2}+2 \mu \omega+\delta^{2}+1=0
$$

Solution of the eikonal equation:

$$
\left(x_{0}+u\right)^{4}+2 \mu\left(x_{0}+u\right)^{3}+\left(\delta^{2}+\mu^{2}+2\right)\left(x_{0}+u\right)^{2}+2 \mu\left(x_{0}+u\right)+\delta^{2}+1=0
$$

(4) $\left\langle 4 X_{4}, P_{1}-X_{2}, P_{2}+X_{1}-\delta X_{3}, \delta>0\right\rangle$.

Ansatz:

$$
x_{3}\left(x_{0}+u\right)^{2}-x_{2} \delta\left(x_{0}+u\right)+\delta x_{1}+x_{3}=\varphi(\omega), \quad \omega=x_{0}+u
$$

Reduced equation:

$$
\left(\omega^{2}+1\right)\left(\omega^{2}+\delta^{2}+1\right)=0
$$

Solutions of the eikonal equation:

$$
\left(x_{0}+u\right)^{2}+1=0,\left(x_{0}+u\right)^{2}+\delta^{2}+1=0
$$

(5) $\left\langle 4 X_{4}, P_{1}-X_{2}-\beta X_{3}, P_{2}+X_{1}, \beta>0\right\rangle$.

Ansatz:

$$
x_{3}\left(x_{0}+u\right)^{2}-\beta x_{1}\left(x_{0}+u\right)-\beta x_{2}+x_{3}=\varphi(\omega), \quad \omega=x_{0}+u
$$

Reduced equation:

$$
\left(\omega^{2}+1\right)\left(\omega^{2}+\beta^{2}+1\right)=0
$$

Solutions of the eikonal equation:

$$
\left(x_{0}+u\right)^{2}+1=0,\left(x_{0}+u\right)^{2}+\beta^{2}+1=0
$$

As we see, the left hand sides of the Ansatzes (1)-(5) are polinomials in invariant $\omega=x_{0}+u$. The reduced equations are also polinomials in variable $\omega$, but with the constant coefficients. The solutions of the eikonal equation are also polinomials in variable $x_{0}+u$ with the constant coefficients.

(6) $\left\langle 4 X_{4}, P_{1}-X_{2}, P_{2}+X_{1}-\mu X_{2}, \mu \neq 0\right\rangle$.

Ansatz:

$$
x_{3}\left(x_{0}+u\right)^{2}+\mu x_{3}\left(x_{0}+u\right)+x_{3}=\varphi(\omega), \quad \omega=x_{0}+u
$$

Reduced equation:

$$
\left(\omega^{2}+\mu \omega+1\right)^{2}=0
$$


Solution of the eikonal equation:

$$
u=-\frac{1}{2}\left(\mu+\left(\mu^{2}-4\right)^{1 / 2}\right)-x_{0}
$$

(7) $\left\langle 2 \mu X_{4}, P_{3}-X_{2}, X_{1}+\mu X_{3}, \mu>0\right\rangle$.

Ansatz:

$$
x_{2}-\frac{x_{3}-\mu x_{1}}{x_{0}+u}=\varphi(\omega), \quad \omega=x_{0}+u
$$

Reduced equation:

$$
\omega^{2}\left(\omega^{2}+\mu^{2}+1\right)=0
$$

Solutions of the reduced equation:

$$
\omega=0, \quad \omega^{2}+\mu^{2}+1=0
$$

Solutions of the eikonal equation:

$$
u=-x_{0},\left(x_{0}+u\right)^{2}+\mu^{2}+1=0
$$

\subsubsection{Reductions to Equations, Which Can Be Split on Two Linear ODEs}

Taking into account the invariants of five nonconjugate subalgebras, we constructed the ansatzes, which reduced the eikonal equation to those, which could be split on two linear ODEs.

(1) $\left\langle 2 \mu X_{4}, P_{3}, X_{1}+\mu X_{3}, \mu>0\right\rangle$.

Ansatz:

$$
x_{0}+u=\varphi(\omega), \quad \omega=x_{2}
$$

Reduced equation:

$$
\left(\varphi^{\prime}\right)^{2}=0
$$

Solution of the reduced equation:

$$
\varphi(\omega)=c_{1}
$$

Solution of the eikonal equation:

$$
u=c_{1}-x_{0}
$$

(2) $\left\langle 2 X_{4}, P_{3}-L_{3}, X_{3}\right\rangle$.

Ansatz:

$$
x_{0}+u=\varphi(\omega), \quad \omega=\left(x_{1}^{2}+x_{2}^{2}\right)^{1 / 2}
$$

Reduced equation:

$$
\left(\varphi^{\prime}\right)^{2}=0
$$

Solution of the reduced equation:

$$
\varphi(\omega)=c_{1}
$$

Solution of the eikonal equation:

$$
u=c_{1}-x_{0}
$$

(3) $\left\langle 2 X_{4}, P_{3}-X_{1}, X_{3}\right\rangle$.

Ansatz:

$$
x_{0}+u=\varphi(\omega), \quad \omega=x_{2}
$$


Reduced equation:

$$
\left(\varphi^{\prime}\right)^{2}=0
$$

Solution of the reduced equation:

$$
\varphi(\omega)=c_{1}
$$

Solution of the eikonal equation:

$$
u=c_{1}-x_{0}
$$

(4) $\left\langle-2 \alpha X_{4}, L_{3}+\alpha X_{3}, P_{3}, \alpha>0\right\rangle$.

Ansatz:

$$
x_{0}+u=\varphi(\omega), \quad \omega=\left(x_{1}^{2}+x_{2}^{2}\right)^{1 / 2}
$$

Reduced equation:

$$
\left(\varphi^{\prime}\right)^{2}=0
$$

Solution of the reduced equation:

$$
\varphi(\omega)=c_{1}
$$

Solution of the eikonal equation:

$$
u=c_{1}-x_{0}
$$

(5) $\left\langle 4 X_{4}, P_{1}-X_{2}, P_{2}+X_{1}\right\rangle$.

Ansatz:

$$
x_{0}+u=\varphi(\omega), \quad \omega=x_{3}
$$

Reduced equation:

$$
\left(\varphi^{\prime}\right)^{2}=0
$$

Solution of the reduced equation:

$$
\varphi(\omega)=c_{1}
$$

Solution of the eikonal equation:

$$
u=c_{1}-x_{0}
$$

Let us note that, in the cases (1)-(5), we obtained the same reduced equation. The solutions of the eikonal equation are also the same.

\subsubsection{Reductions to Nonlinear ODEs}

From the invariants of four nonconjugate subalgebras, we constructed the ansatzes, which reduced the eikonal equation to nonlinear ODEs.

(1) $\left\langle 2 \mu X_{4}, P_{3}-2 X_{0}, X_{1}+\mu X_{3}, \mu>0\right\rangle$.

Ansatz:

$$
\left(x_{0}+u\right)^{2}+4 x_{3}-4 \mu x_{1}=\varphi(\omega), \quad \omega=x_{2}
$$

Reduced equation:

$$
\left(\varphi^{\prime}\right)^{2}+16\left(\mu^{2}+1\right)=0
$$

Solutions of the reduced equation:

$$
\varphi(\omega)=4 i \varepsilon\left(\mu^{2}+1\right)^{1 / 2} \omega+c_{1}, \varepsilon= \pm 1
$$


Solutions of the eikonal equation:

$$
u=2\left(i \varepsilon x_{2} \sqrt{\mu^{2}+1}+\mu x_{1}-x_{3}+c_{1}\right)^{1 / 2}-x_{0}, \varepsilon= \pm 1
$$

(2) $\left\langle 2 X_{4}, P_{3}-L_{3}-2 \alpha X_{0}, X_{3}, \alpha>0\right\rangle$.

Ansatz:

$$
2 \alpha \arctan \frac{x_{1}}{x_{2}}-x_{0}-u=\varphi(\omega), \quad \omega=\left(x_{1}^{2}+x_{2}^{2}\right)^{1 / 2}
$$

Reduced equation:

$$
\omega^{2}\left(\varphi^{\prime}\right)^{2}+4 \alpha^{2}=0
$$

Solutions of the reduced equation:

$$
\varphi(\omega)=2 i \varepsilon \alpha \ln (\omega)+c_{1}, \varepsilon= \pm 1
$$

Solutions of the eikonal equation:

$$
u=2 \alpha \arctan \frac{x_{1}}{x_{2}}+i \varepsilon \alpha \ln \left(x_{1}^{2}+x_{2}^{2}\right)-x_{0}+c_{1}, \varepsilon= \pm 1
$$

(3) $\left\langle-2 \beta X_{4}, L_{3}+\beta X_{3}, P_{3}-2 X_{0}, \beta>0\right\rangle$.

Ansatz:

$$
\beta \arctan \frac{x_{1}}{x_{2}}+\frac{1}{4}\left(x_{0}+u\right)^{2}+x_{3}=\varphi(\omega), \quad \omega=\left(x_{1}^{2}+x_{2}^{2}\right)^{1 / 2}
$$

Reduced equation:

$$
\omega^{2}\left(\varphi^{\prime}\right)^{2}+\omega^{2}+\beta^{2}=0
$$

Solutions of the reduced equation:

$$
\varphi(\omega)=i \varepsilon \sqrt{\omega^{2}+\beta^{2}}-i \varepsilon \beta \operatorname{artanh} \frac{\beta}{\sqrt{\omega^{2}+\beta^{2}}}+c_{1}, \varepsilon= \pm 1
$$

Solutions of the eikonal equation:

$$
\beta \arctan \frac{x_{1}}{x_{2}}+\frac{1}{4}\left(x_{0}+u\right)^{2}=i \varepsilon \sqrt{x_{1}^{2}+x_{2}^{2}+\beta^{2}}-i \varepsilon \beta \operatorname{artanh} \frac{\beta}{\sqrt{x_{1}^{2}+x_{2}^{2}+\beta^{2}}}-x_{3}+c_{1}, \varepsilon= \pm 1
$$

(4) $\left\langle 2 X_{4}, P_{3}, X_{3}\right\rangle$.

Ansatz:

$$
x_{2}=\varphi\left(\omega_{1}, \omega_{2}\right), \quad \omega_{1}=x_{0}+u, \omega_{2}=x_{1} .
$$

Reduced equation:

$$
\varphi_{2}^{2}+1=0
$$

Solution of the reduced equation:

$$
\varphi\left(\omega_{1}, \omega_{2}\right)=i \omega_{2}+f\left(\omega_{1}\right)
$$

where $f$ is an arbitrary function.

Solution of the eikonal equation:

$$
x_{2}=i x_{1}+f\left(x_{0}+u\right)
$$


where $f$ is an arbitrary function.

\subsubsection{There Are No Reductions}

From the invariants of remaining one nonconjugate subalgebra, it is impossible to construct the ansatz, which reduces the eikonal equation.

Below, we present the subalgebra as well as its invariants.

$$
\left\langle 2 X_{4}, P_{3}-2 X_{0}, X_{3}\right\rangle: x_{1}, x_{2}
$$

3.4. Lie Algebras of the Type $A_{3,2}$

$$
\left[e_{1}, e_{3}\right]=e_{1},\left[e_{2}, e_{3}\right]=e_{1}+e_{2}
$$

The Lie algebras of the type $A_{3,2}$ are solvable $[41,43]$.

The Lie algebra of the group $P(1,4)$ contains three nonconjugate subalgebras of the type $A_{3,2}$. Below, we present the results obtained for those subalgebras.

\subsubsection{Reductions to Nonlinear ODEs}

From the invariants of two nonconjugate subalgebras, we constructed the ansatzes, which reduced the eikonal equation to nonlinear ODEs.

(1) $\left\langle 2 \beta X_{4}, P_{3}, G+\alpha X_{1}+\beta X_{3}, \alpha>0, \beta>0\right\rangle$.

Ansatz:

$$
x_{1}-\alpha \ln \left(x_{0}+u\right)=\varphi(\omega), \quad \omega=x_{2}
$$

Reduced equation:

$$
\left(\varphi^{\prime}\right)^{2}+1=0
$$

Solutions of the reduced equation:

$$
\varphi(\omega)=i \varepsilon \omega+c_{1}, \varepsilon= \pm 1
$$

Solutions of the eikonal equation:

$$
x_{1}-\alpha \ln \left(x_{0}+u\right)=i \varepsilon x_{2}+c_{1}, \varepsilon= \pm 1
$$

(2) $\left\langle 2 \alpha X_{4}, \lambda P_{3}, \frac{1}{\lambda} L_{3}+G+\frac{\alpha}{\lambda} X_{3}, \alpha>0, \lambda>0\right\rangle$.

Ansatz:

$$
\ln \left(x_{0}+u\right)+\lambda \arctan \frac{x_{1}}{x_{2}}=\varphi(\omega), \quad \omega=\left(x_{1}^{2}+x_{2}^{2}\right)^{1 / 2}
$$

Reduced equation:

$$
\omega^{2}\left(\varphi^{\prime}\right)^{2}+\lambda^{2}=0
$$

Solutions of the reduced equation:

$$
\varphi(\omega)=i \varepsilon \lambda \ln (\omega)+c_{1}, \varepsilon= \pm 1
$$

Solutions of the eikonal equation:

$$
\ln \left(x_{0}+u\right)+\lambda \arctan \frac{x_{1}}{x_{2}}=i \varepsilon \frac{\lambda}{2} \ln \left(x_{1}^{2}+x_{2}^{2}\right)+c_{1}, \varepsilon= \pm 1
$$




\subsubsection{There Are No Reductions}

From the invariants of remaining one nonconjugate subalgebra, it is impossible to construct the ansatz, which reduces the eikonal equation.

Below, we present the subalgebra as well as its invariants.

$$
\left\langle 2 \alpha X_{4}, P_{3}, G+\alpha X_{3}, \alpha>0\right\rangle: x_{1}, x_{2}
$$

3.5. Lie Algebras of the Type $A_{3,3}$

$$
\left[e_{1}, e_{3}\right]=e_{1},\left[e_{2}, e_{3}\right]=e_{2}
$$

The Lie algebras of the type $A_{3,3}$ are solvable [41,43].

The Lie algebra of the group $P(1,4)$ contains five nonconjugate subalgebras of the type $A_{3,3}$. Below, we present the results obtained for those subalgebras.

\subsubsection{Reductions to Equations, Which Can Be Split on Two Linear ODEs}

Taking into account the invariants of one nonconjugate subalgebra, we constructed the ansatz, which reduced the eikonal equation to those, which could be split on two linear ODEs.

$$
\left\langle P_{1}, P_{2}, G\right\rangle
$$

Ansatz:

$$
\left(x_{0}^{2}-x_{1}^{2}-x_{2}^{2}-u^{2}\right)^{1 / 2}=\varphi(\omega), \quad \omega=x_{3}
$$

Reduced equation:

$$
\left(\varphi^{\prime}-1\right)\left(\varphi^{\prime}+1\right) \varphi^{2}=0
$$

Solutions of the reduced equation:

$$
\varphi(\omega)=\varepsilon \omega+c, \varepsilon= \pm 1, \quad \varphi=0
$$

Solutions of the eikonal equation:

$$
\left(x_{0}^{2}-x_{1}^{2}-x_{2}^{2}-u^{2}\right)^{1 / 2}=\varepsilon x_{3}+c_{1}, \varepsilon= \pm 1, \quad x_{0}^{2}-x_{1}^{2}-x_{2}^{2}-u^{2}=0
$$

\subsubsection{Reductions to Nonlinear ODEs}

From the invariants of three subalgebras, we constructed the ansatzes, which reduced the eikonal equation to nonlinear ODEs.

(1) $\left\langle P_{1}, P_{2}, G+\alpha X_{3}, \alpha>0\right\rangle$.

Ansatz:

$$
x_{3}-\alpha \ln \left(x_{0}+u\right)=\varphi(\omega), \quad \omega=x_{0}^{2}-x_{1}^{2}-x_{2}^{2}-u^{2}
$$

The reduced equation:

$$
4 \omega\left(\varphi^{\prime}\right)^{2}+4 \alpha \varphi^{\prime}-1=0
$$

Solutions of the reduced equation:

$$
\varphi(\omega)=\varepsilon\left(\alpha^{2}+\omega\right)^{1 / 2}-i \varepsilon \alpha \arctan \frac{\left(\alpha^{2}+\omega\right)^{1 / 2}}{i \alpha}-\frac{\alpha}{2} \ln \omega+c, \quad \varepsilon= \pm 1
$$


Solutions of the eikonal equation:

$$
\begin{aligned}
x_{3}-\alpha \ln \left(x_{0}+u\right)= & \varepsilon\left(x_{0}^{2}-x_{1}^{2}-x_{2}^{2}-u^{2}+\alpha^{2}\right)^{1 / 2}-i \varepsilon \alpha \arctan \frac{\left(x_{0}^{2}-x_{1}^{2}-x_{2}^{2}-u^{2}+\alpha^{2}\right)^{1 / 2}}{i \alpha}- \\
& -\frac{\alpha}{2} \ln \left(x_{0}^{2}-x_{1}^{2}-x_{2}^{2}-u^{2}\right)+c, \quad \varepsilon= \pm 1
\end{aligned}
$$

(2) $\left\langle P_{3}, X_{4}, \frac{1}{\lambda} L_{3}+G, \lambda>0\right\rangle$.

Ansatz:

$$
\ln \left(x_{0}+u\right)+\lambda \arctan \frac{x_{1}}{x_{2}}=\varphi(\omega), \quad \omega=\left(x_{1}^{2}+x_{2}^{2}\right)^{1 / 2}
$$

Reduced equation:

$$
\omega^{2}\left(\varphi^{\prime}\right)^{2}+\lambda^{2}=0
$$

Solutions of the reduced equation:

$$
\varphi(\omega)=i \varepsilon \lambda \ln (\omega)+c, \varepsilon= \pm 1
$$

Solutions of the eikonal equation:

$$
\ln \left(x_{0}+u\right)+\lambda \arctan \frac{x_{1}}{x_{2}}=i \varepsilon \frac{\lambda}{2} \ln \left(x_{1}^{2}+x_{2}^{2}\right)+c, \varepsilon= \pm 1
$$

(3) $\left\langle P_{3}, X_{4}, G+\alpha X_{1}, \alpha>0\right\rangle$.

Ansatz:

$$
x_{1}-\alpha \ln \left(x_{0}+u\right)=\varphi(\omega), \quad \omega=x_{2}
$$

Reduced equation:

$$
\left(\varphi^{\prime}\right)^{2}+1=0
$$

Solutions of the reduced equation:

$$
\varphi(\omega)=i \varepsilon \omega+c, \quad \varepsilon= \pm 1
$$

Solutions of the eikonal equation:

$$
x_{1}-\alpha \ln \left(x_{0}+u\right)=i \varepsilon x_{2}+c_{1}, \quad \varepsilon= \pm 1
$$

\subsubsection{There Are No Reductions}

From the invariants of remaining one nonconjugate subalgebra, it is impossible to construct the ansatz, which reduces the eikonal equation.

Below, we present the subalgebra as well as its invariants.

$$
\left\langle P_{3}, X_{4}, G\right\rangle: x_{1}, x_{2}
$$

3.6. Lie Algebras of the Type $A_{3,4}$

$$
\left[e_{1}, e_{3}\right]=e_{1},\left[e_{2}, e_{3}\right]=-e_{2}
$$

The Lie algebras of the type $A_{3,4}$ are solvable $[41,43]$.

The Lie algebra of the group $P(1,4)$ contains four nonconjugate subalgebras of the type $A_{3,4}$. 


\subsubsection{There Are No Reductions}

From the invariants of all four nonconjugate subalgebras, it is impossible to construct the ansatzes, which reduce the eikonal equation.

Below, we present those subalgebras as well as their invariants.

(1) $\left\langle X_{4}, X_{0}, G\right\rangle: x_{1}, x_{2}, x_{3}$;

(2) $\left\langle X_{0},-X_{4},-\frac{1}{\lambda} L_{3}-G, \lambda>0\right\rangle: x_{3},\left(x_{1}^{2}+x_{2}^{2}\right)^{1 / 2}$;

(3) $\left\langle X_{0}, X_{4},-\left(G+\alpha X_{1}\right), \alpha>0\right\rangle: x_{2}, x_{3}$;

(4) $\left\langle X_{0}, X_{4},-\frac{L_{3}}{\lambda}-G-\frac{\alpha}{\lambda} X_{3}, \alpha>0, \lambda>0\right\rangle:\left(x_{1}^{2}+x_{2}^{2}\right)^{1 / 2}, x_{3}+\alpha \arctan \frac{x_{1}}{x_{2}}$.

3.7. Lie Algebras of the Type $A_{3,5}^{a}$

$$
\left[e_{1}, e_{3}\right]=e_{1},\left[e_{2}, e_{3}\right]=a e_{2},(0<|a|<1)
$$

The Lie algebra of the group $P(1,4)$ contains no nonconjugate subalgebras of the type $A_{3,5}^{a}$.

3.8. Lie Algebras of the Type $A_{3,6}$

$$
\left[e_{1}, e_{3}\right]=-e_{2},\left[e_{2}, e_{3}\right]=e_{1}
$$

The Lie algebras of the type $A_{3,6}$ are solvable [41,43].

The Lie algebra of the group $P(1,4)$ contains 18 nonconjugate subalgebras of the type $A_{3,6}$.

Below, we present the results obtained for those subalgebras.

\subsubsection{Reductions to Linear ODEs}

The invariants of four subalgebras allow us to construct the ansatzes, which reduce the eikonal equation to linear ODEs.

(1) $\left\langle P_{1}-X_{1}, P_{2}-X_{2},-P_{3}+L_{3}\right\rangle$.

Ansatz:

$$
\frac{x_{1}^{2}+x_{2}^{2}}{x_{0}+u+1}+\frac{x_{3}^{2}}{x_{0}+u}+2 u=\varphi(\omega), \quad \omega=x_{0}+u
$$

Reduced equation:

$$
\omega^{4}(\omega+1)^{4}\left(\varphi^{\prime}-1\right)=0
$$

Solutions of the reduced equation:

$$
\omega+1=0, \quad \omega=0, \quad \varphi(\omega)=\omega+c_{1}
$$

Solutions of the eikonal equation:

$$
u=-1-x_{0}, u=-x_{0}, \quad \frac{x_{1}^{2}+x_{2}^{2}}{x_{0}+u+1}+\frac{x_{3}^{2}}{x_{0}+u}+2 u=x_{0}+u+c_{1}
$$

(2) $\left\langle P_{1},-P_{2},-\left(L_{3}+\alpha X_{3}\right), \alpha>0\right\rangle$.

Ansatz:

$$
x_{0}^{2}-x_{1}^{2}-x_{2}^{2}-u^{2}=\varphi(\omega), \quad \omega=x_{0}+u
$$

Reduced equation:

$$
\omega \varphi^{\prime}-\varphi=0
$$


Solution of the reduced equation:

$$
\varphi(\omega)=c \omega
$$

Solution of the eikonal equation:

$$
x_{0}^{2}-x_{1}^{2}-x_{2}^{2}-u^{2}=c\left(x_{0}+u\right)
$$

(3) $\left\langle X_{1},-X_{2}, P_{3}-L_{3}\right\rangle$.

Ansatz:

$$
x_{0}^{2}-x_{3}^{2}-u^{2}=\varphi(\omega), \quad \omega=x_{0}+u
$$

Reduced equation:

$$
\omega \varphi^{\prime}-\varphi=0
$$

Solution of the reduced equation:

$$
\varphi(\omega)=c_{1} \omega
$$

Solution of the eikonal equation:

$$
x_{0}^{2}-x_{3}^{2}-u^{2}=c_{1}\left(x_{0}+u\right)
$$

(4) $\left\langle P_{1}, P_{2},-P_{3}+L_{3}\right\rangle$.

Ansatz:

$$
x_{0}^{2}-x_{1}^{2}-x_{2}^{2}-x_{3}^{2}-u^{2}=\varphi(\omega), \quad \omega=x_{0}+u
$$

Reduced equation:

$$
\omega \varphi^{\prime}-\varphi=0
$$

Solution of the reduced equation:

$$
\varphi(\omega)=c_{1} \omega
$$

Solution of the eikonal equation:

$$
x_{0}^{2}-x_{1}^{2}-x_{2}^{2}-x_{3}^{2}-u^{2}=c_{1}\left(x_{0}+u\right)
$$

\subsubsection{Reductions to Equations, Which Can Be Split on Two Linear ODEs}

Taking into account the invariants of seven nonconjugate subalgebras, we constructed the ansatzes, which reduced the eikonal equation to those, which could be split on two linear ODEs.

(1) $\left\langle X_{1},-X_{2},-\left(L_{3}+2 X_{4}\right)\right\rangle$.

Ansatz:

$$
x_{0}+u=\varphi(\omega), \quad \omega=x_{3}
$$

Reduced equation:

$$
\left(\varphi^{\prime}\right)^{2}=0
$$

Solution of the reduced equation:

$$
\varphi(\omega)=c_{1}
$$

Solution of the eikonal equation:

$$
u=c_{1}-x_{0}
$$


(2) $\left\langle P_{1}, P_{2}, L_{3}+2 X_{4}\right\rangle$.

Ansatz:

$$
x_{0}+u=\varphi(\omega), \quad \omega=x_{3}
$$

Reduced equation:

$$
\left(\varphi^{\prime}\right)^{2}=0
$$

Solution of the reduced equation:

$$
\varphi(\omega)=c_{1}
$$

Solution of the eikonal equation:

$$
u=c_{1}-x_{0}
$$

(3) $\left\langle X_{1}, X_{2}, L_{3}+\frac{1}{2}\left(P_{3}+C_{3}\right)\right\rangle$.

Ansatz:

$$
\left(x_{3}^{2}+u^{2}\right)^{1 / 2}=\varphi(\omega), \quad \omega=x_{0}
$$

Reduced equation:

$$
\left(\varphi^{\prime}-1\right)\left(\varphi^{\prime}+1\right) \varphi^{2}=0
$$

Solutions of the reduced equation:

$$
\varphi(\omega)=\varepsilon \omega+c_{1}, \varepsilon= \pm 1, \quad \varphi=0
$$

Solutions of the eikonal equation:

$$
\left(x_{3}^{2}+u^{2}\right)^{1 / 2}=\varepsilon x_{0}+c, \varepsilon= \pm 1, \quad x_{3}^{2}+u^{2}=0
$$

(4) $\left\langle-X_{1}, X_{2},-L_{3}-\frac{\lambda}{2}\left(P_{3}+C_{3}\right), 0<\lambda<1\right\rangle$.

Ansatz:

$$
\left(x_{3}^{2}+u^{2}\right)^{1 / 2}=\varphi(\omega), \quad \omega=x_{0}
$$

Reduced equation:

$$
\left(\varphi^{\prime}-1\right)\left(\varphi^{\prime}+1\right) \varphi^{2}=0
$$

Solutions of the reduced equation:

$$
\varphi(\omega)=\varepsilon \omega+c_{1}, \varepsilon= \pm 1, \quad \varphi=0
$$

Solutions of the eikonal equation:

$$
\left(x_{3}^{2}+u^{2}\right)^{1 / 2}=\varepsilon x_{0}+c_{1}, \varepsilon= \pm 1, \quad x_{3}^{2}+u^{2}=0
$$

(5) $\left\langle-X_{1}, X_{2},-\left(L_{3}+\lambda G\right), \lambda>0\right\rangle$.

Ansatz:

$$
\left(x_{0}^{2}-u^{2}\right)^{1 / 2}=\varphi(\omega), \quad \omega=x_{3}
$$

Reduced equation:

$$
\left(\varphi^{\prime}-1\right)\left(\varphi^{\prime}+1\right)=0
$$

Solutions of the reduced equation:

$$
\varphi(\omega)=\varepsilon \omega+c_{1}, \varepsilon= \pm 1
$$


Solutions of the eikonal equation:

$$
\left(x_{0}^{2}-u^{2}\right)^{1 / 2}=\varepsilon x_{3}+c_{1}, \varepsilon= \pm 1
$$

(6) $\left\langle X_{1},-X_{2},-\left(L_{3}+\alpha X_{3}\right), \alpha>0\right\rangle$.

Ansatz:

$$
u=\varphi(\omega), \quad \omega=x_{0}
$$

Reduced equation:

$$
\left(\varphi^{\prime}-1\right)\left(\varphi^{\prime}+1\right)=0
$$

Solutions of the reduced equation:

$$
\varphi(\omega)=\varepsilon \omega+c_{1}, \varepsilon= \pm 1
$$

Solutions of the eikonal equation:

$$
u=\varepsilon x_{0}+c_{1}, \varepsilon= \pm 1
$$

(7) $\left\langle X_{1},-X_{2}, P_{3}-L_{3}-2 \alpha X_{0}, \alpha>0\right\rangle$.

Ansatz:

$$
\left(x_{0}+u\right)^{3}+6 \alpha x_{3}\left(x_{0}+u\right)+6 \alpha^{2}\left(x_{0}-u\right)=\varphi(\omega), \quad \omega=\left(x_{0}+u\right)^{2}+4 x_{3} \alpha
$$

Reduced equation:

$$
4\left(\varphi^{\prime}\right)^{2}-9 \omega=0
$$

Solutions of the reduced equation:

$$
\varphi(\omega)=\varepsilon \omega^{3 / 2}+c, \varepsilon= \pm 1
$$

Solutions of the eikonal equation:

$$
\left(x_{0}+u\right)^{3}+6 \alpha x_{3}\left(x_{0}+u\right)+6 \alpha^{2}\left(x_{0}-u\right)=\varepsilon\left(\left(x_{0}+u\right)^{2}+4 \alpha x_{3}\right)^{3 / 2}+c_{1}, \varepsilon= \pm 1
$$

\subsubsection{Reductions to Nonlinear ODEs}

From the invariants of three nonconjugate subalgebras, we constructed the ansatzes, which reduced the eikonal equation to nonlinear ODEs.

(1) $\left\langle X_{1},-X_{2},-L_{3}-\frac{1}{2}\left(P_{3}+C_{3}\right)-\alpha\left(X_{0}+X_{4}\right), \alpha>0\right\rangle$.

Ansatz:

$$
\alpha \arctan \frac{x_{3}}{u}-x_{0}=\varphi(\omega), \quad \omega=\left(x_{3}^{2}+u^{2}\right)^{1 / 2}
$$

Reduced equation:

$$
\omega^{4}\left(\omega^{2}\left(\varphi^{\prime}\right)^{2}-\omega^{2}+\alpha^{2}\right)=0
$$

Solutions of the reduced equation:

$$
\omega=0, \quad \varphi(\omega)=\varepsilon \sqrt{\omega^{2}-\alpha^{2}}-i \varepsilon \alpha \operatorname{artanh} \frac{i \alpha}{\sqrt{\omega^{2}-\alpha^{2}}}+c, \varepsilon= \pm 1
$$


Solutions of the eikonal equation:

$$
x_{3}^{2}+u^{2}=0, \alpha \arctan \frac{x_{3}}{u}-x_{0}=\varepsilon \sqrt{x_{3}^{2}+u^{2}-\alpha^{2}}-i \varepsilon \alpha \operatorname{artanh} \frac{i \alpha}{\sqrt{x_{3}^{2}+u^{2}-\alpha^{2}}}+c, \varepsilon= \pm 1
$$

(2) $\left\langle X_{1}, X_{2}, L_{3}+\frac{\lambda}{2}\left(P_{3}+C_{3}\right)+\alpha\left(X_{0}+X_{4}\right), \alpha>0,0<\lambda<1\right\rangle$.

Ansatz:

$$
\alpha \arctan \frac{x_{3}}{u}-\lambda x_{0}=\varphi(\omega), \quad \omega=\left(x_{3}^{2}+u^{2}\right)^{1 / 2}
$$

Reduced equation:

$$
\omega^{4}\left(\omega^{2}\left(\varphi^{\prime}\right)^{2}-\lambda^{2} \omega^{2}+\alpha^{2}\right)=0
$$

Solutions of the reduced equation:

$$
\omega=0, \quad \varphi(\omega)=\varepsilon \sqrt{\lambda^{2} \omega^{2}-\alpha^{2}}-i \varepsilon \alpha \operatorname{artanh} \frac{i \alpha}{\sqrt{\lambda^{2} \omega^{2}-\alpha^{2}}}+c, \varepsilon= \pm 1
$$

Solutions of the eikonal equation:

$$
\begin{gathered}
\alpha \arctan \frac{x_{3}}{u}-\lambda x_{0}=\varepsilon \sqrt{\lambda^{2}\left(x_{3}^{2}+u^{2}\right)-\alpha^{2}}-i \varepsilon \alpha \operatorname{artanh} \frac{i \alpha}{\sqrt{\lambda^{2}\left(x_{3}^{2}+u^{2}\right)-\alpha^{2}}}+c \\
\varepsilon= \pm 1, \quad x_{3}^{2}+u^{2}=0
\end{gathered}
$$

(3) $\left\langle X_{1}, X_{2}, L_{3}+\lambda G+\alpha X_{3}, \alpha>0, \lambda>0\right\rangle$.

Ansatz:

$$
\lambda x_{3}-\alpha \ln \left(x_{0}+u\right)=\varphi(\omega), \quad \omega=\left(x_{0}^{2}-u^{2}\right)^{1 / 2}
$$

Reduced equation:

$$
\omega\left(\omega\left(\varphi^{\prime}\right)^{2}+2 \alpha \varphi^{\prime}-\lambda^{2} \omega\right)=0
$$

Solutions of the reduced equation:

$$
\omega=0, \varphi(\omega)=\varepsilon \sqrt{\lambda^{2} \omega^{2}+\alpha^{2}}-\varepsilon \alpha \operatorname{artanh} \frac{\alpha}{\sqrt{\lambda^{2} \omega^{2}+\alpha^{2}}}-\alpha \ln (\omega)+c_{1}, \varepsilon= \pm 1 .
$$

Solutions of the eikonal equation:

$$
\begin{gathered}
x_{0}^{2}-u^{2}=0 \\
\lambda x_{3}-\alpha \ln \left(x_{0}+u\right)=\varepsilon \sqrt{\lambda^{2}\left(x_{0}^{2}-u^{2}\right)+\alpha^{2}}-\varepsilon \alpha \operatorname{artanh} \frac{\alpha}{\sqrt{\lambda^{2}\left(x_{0}^{2}-u^{2}\right)+\alpha^{2}}}-\frac{\alpha}{2} \ln \left(x_{0}^{2}-u^{2}\right)+c_{1} \\
\varepsilon= \pm 1
\end{gathered}
$$

\subsubsection{Reductions to PDEs}

From the invariants of two nonconjugate subalgebras, we constructed the ansatzes, which reduced the eikonal equation to PDEs.

(1) $\left\langle X_{1}, X_{2}, L_{3}\right\rangle$.

Ansatz:

$$
u=\varphi\left(\omega_{1}, \omega_{2}\right), \quad \omega_{1}=x_{0}, \omega_{2}=x_{3}
$$

Reduced equation:

$$
\varphi_{1}^{2}-\varphi_{2}^{2}-1=0
$$


Solutions of the reduced equation:

$$
\varphi\left(\omega_{1}, \omega_{2}\right)=\varepsilon \sqrt{c_{2}^{2}+1} \omega_{1}+c_{2} \omega_{2}+c_{1}+c_{2}, \varepsilon= \pm 1
$$

Solutions of the eikonal equation:

$$
u=\varepsilon \sqrt{c_{2}^{2}+1} x_{0}+c_{2} x_{3}+c_{1}+c_{2}, \varepsilon= \pm 1
$$

(2) $\left\langle P_{1}, P_{2}, L_{3}\right\rangle$.

Ansatz:

$$
x_{3}=\varphi\left(\omega_{1}, \omega_{2}\right), \quad \omega_{1}=x_{0}+u, \omega_{2}=x_{0}^{2}-x_{1}^{2}-x_{2}^{2}-u^{2}
$$

The reduced equation:

$$
4 \omega_{2} \varphi_{2}^{2}+4 \omega_{1} \varphi_{1} \varphi_{2}-1=0
$$

Solutions of the reduced equation:

$$
\varphi\left(\omega_{1}, \omega_{2}\right)=c_{1} \ln \omega_{1}-\varepsilon\left(\omega_{2}+c_{1}^{2}\right)^{1 / 2}+i \varepsilon c_{1} \arctan \frac{\sqrt{\omega_{2}+c_{1}^{2}}}{i c_{1}}-\frac{c_{1}}{2} \ln \omega_{2}+c_{2}, \varepsilon= \pm 1
$$

Solutions on the eikonal equation:

$$
\begin{gathered}
x_{3}=c_{1} \ln \left(x_{0}+u\right)-\varepsilon\left(x_{0}^{2}-x_{1}^{2}-x_{2}^{2}-u^{2}+c_{1}^{2}\right)^{1 / 2} \\
+i \varepsilon c_{1} \arctan \frac{\sqrt{x_{0}^{2}-x_{1}^{2}-x_{2}^{2}-u^{2}+c_{1}^{2}}}{i c_{1}} \\
-\frac{c_{1}}{2} \ln \left(x_{0}^{2}-x_{1}^{2}-x_{2}^{2}-u^{2}\right)+c_{2} \\
\varepsilon= \pm 1
\end{gathered}
$$

As we see, in the above two cases, the reduced equations are PDEs. The reason is that the subalgebras corresponding to them have the rank two. Therefore, they have three invariants. As a rule, the ansatzes, which can be constructed with the help of those invariants, reduce the eikonal equation to PDEs.

\subsubsection{There Are No Reductions}

From the invariants of remaining two nonconjugate subalgebras, it is impossible to construct the ansatzes, which reduce the eikonal equation.

Below, we present those subalgebras as well as their invariants.

(1) $\left\langle-X_{3}, X_{4}-X_{0},-\frac{L_{3}}{\lambda}-\frac{1}{2}\left(P_{3}+C_{3}\right), 0<\lambda<1\right\rangle: x_{0},\left(x_{1}^{2}+x_{2}^{2}\right)^{1 / 2}$

(2) $\left\langle X_{3}, X_{4}-X_{0}, \frac{L_{3}}{\lambda}+\frac{1}{2}\left(P_{3}+C_{3}\right)+\frac{\alpha}{\lambda}\left(X_{0}+X_{4}\right), \alpha>0,0<\lambda<1\right\rangle:\left(x_{1}^{2}+x_{2}^{2}\right)^{1 / 2}$, $\alpha \arctan \frac{x_{1}}{x_{2}}-x_{0}$.

3.9. Lie Algebras of the Type $A_{3,7}^{a}$

$$
\left[e_{1}, e_{3}\right]=a e_{1}-e_{2},\left[e_{2}, e_{3}\right]=e_{1}+a e_{2},(a>0) . \quad a=c:
$$

The Lie algebras of the type $A_{3,7}^{a}$ are solvable $[41,43]$. 
The Lie algebra of the group $P(1,4)$ contains two nonconjugate subalgebras of the type $A_{3,7}^{a}$. Below, we present the results obtained for those subalgebras.

\subsubsection{Reductions to Equations, Which Can Be Split on Two Linear ODEs}

Taking into account the invariants of one nonconjugate subalgebra, we constructed the ansatz, which reduced the eikonal equation to those, which could be split on two linear ODEs.

$$
\left\langle P_{1}, P_{2}, L_{3}+\lambda G, \lambda>0\right\rangle
$$

Ansatz:

$$
\left(x_{0}^{2}-x_{1}^{2}-x_{2}^{2}-u^{2}\right)^{1 / 2}=\varphi(\omega), \quad \omega=x_{3}
$$

Reduced equation:

$$
\left(\varphi^{\prime}-1\right)\left(\varphi^{\prime}+1\right) \varphi^{2}=0
$$

Solutions of the reduced equation:

$$
\varphi(\omega)=\varepsilon \omega+c_{1}, \varepsilon= \pm 1, \quad \varphi=0
$$

Solutions of the eikonal equation:

$$
\left(x_{0}^{2}-x_{1}^{2}-x_{2}^{2}-u^{2}\right)^{1 / 2}=\varepsilon x_{3}+c_{1}, \varepsilon= \pm 1, \quad x_{0}^{2}-x_{1}^{2}-x_{2}^{2}-u^{2}=0
$$

\subsubsection{Reductions to Nonlinear ODEs}

From the invariants of one nonconjugate subalgebra, we constructed the ansatz, which reduced the eikonal equation to nonlinear ODEs.

$$
\left\langle P_{1}, P_{2}, L_{3}+\lambda G+\alpha X_{3}, \alpha>0, \lambda>0\right\rangle
$$

Ansatz:

$$
\lambda x_{3}-\alpha \ln \left(x_{0}+u\right)=\varphi(\omega), \quad \omega=x_{0}^{2}-x_{1}^{2}-x_{2}^{2}-u^{2}
$$

The reduced equation:

$$
4 \omega\left(\varphi^{\prime}\right)^{2}+4 \alpha \varphi^{\prime}-\lambda^{2}=0
$$

Solutions of the reduced equation:

$$
\varphi(\omega)=\varepsilon\left(\lambda^{2} \omega+\alpha^{2}\right)^{1 / 2}-i \varepsilon \alpha \arctan \frac{\sqrt{\lambda^{2} \omega+\alpha^{2}}}{i \alpha}-\frac{\alpha}{2} \ln \omega+c, \quad \varepsilon= \pm 1
$$

Solutions of the eikonal equation:

$$
\begin{gathered}
\lambda x_{3}-\alpha \ln \left(x_{0}+u\right)=\varepsilon\left(\lambda^{2}\left(x_{0}^{2}-x_{1}^{2}-x_{2}^{2}-u^{2}\right)+\alpha^{2}\right)^{1 / 2} \\
-i \varepsilon \alpha \arctan \frac{\sqrt{\lambda^{2}\left(x_{0}^{2}-x_{1}^{2}-x_{2}^{2}-u^{2}\right)+\alpha^{2}}}{i \alpha} \\
-\frac{\alpha}{2} \ln \left(x_{0}^{2}-x_{1}^{2}-x_{2}^{2}-u^{2}\right)+c \\
\varepsilon= \pm 1
\end{gathered}
$$

3.10. Lie Algebras of the Type $A_{3,8}$

$$
\left[e_{1}, e_{3}\right]=-2 e_{2},\left[e_{1}, e_{2}\right]=e_{1},\left[e_{2}, e_{3}\right]=e_{3}
$$

The Lie algebras of the type $A_{3,8}$ are semisimple [43]. 
The Lie algebra of the group $P(1,4)$ contains one nonconjugate subalgebra of the type $A_{3,8}$. Below, we present the results obtained for this subalgebra.

\subsubsection{Reductions to PDEs}

From the invariants of one nonconjugate subalgebra, we constructed the ansatz, which reduced the eikonal equation to PDEs.

$$
\left\langle P_{3}, G,-C_{3}\right\rangle
$$

Ansatz:

$$
\left(x_{0}^{2}-x_{3}^{2}-u^{2}\right)^{1 / 2}=\varphi\left(\omega_{1}, \omega_{2}\right), \quad \omega_{1}=x_{1}, \omega_{2}=x_{2}
$$

Reduced equation:

$$
\varphi^{2}\left(\varphi_{1}^{2}+\varphi_{2}^{2}-1\right)=0
$$

Solutions of the reduced equation:

$$
\varphi\left(\omega_{1}, \omega_{2}\right)=0, \quad \varphi\left(\omega_{1}, \omega_{2}\right)=\varepsilon \sqrt{1-c_{2}^{2}} \omega_{1}+c_{2} \omega_{2}+c_{1}+c_{2}, \varepsilon= \pm 1
$$

Solutions of the eikonal equation:

$$
x_{0}^{2}-x_{3}^{2}-u^{2}=0,\left(x_{0}^{2}-x_{3}^{2}-u^{2}\right)^{1 / 2}=\varepsilon\left(1-c_{2}^{2}\right)^{1 / 2} x_{1}+c_{2} x_{2}+c_{1}+c_{2}, \varepsilon= \pm 1
$$

As we see, the reduced equation is PDEs. As above, the reason is that the corresponding subalgebra has rank two.

3.11. Lie Algebras of the Type $A_{3,9}$

$$
\left[e_{1}, e_{2}\right]=e_{3},\left[e_{2}, e_{3}\right]=e_{1},\left[e_{3}, e_{1}\right]=e_{2}
$$

The Lie algebras of the type $A_{3,9}$ are semisimple [43].

The Lie algebra of the group $P(1,4)$ contains two nonconjugate subalgebras of the type $A_{3,9}$. Below, we present the results obtained for those subalgebras.

\subsubsection{Reductions to Equations, Which Can Be Split on Two Linear ODEs}

Taking into account the invariants of one nonconjugate subalgebra, we constructed the ansatz, which reduced the eikonal equation to those, which could be split on two linear ODEs.

$$
\left\langle-\frac{1}{2}\left(L_{3}+\frac{1}{2}\left(P_{3}+C_{3}\right)\right), \frac{1}{2}\left(L_{2}+\frac{1}{2}\left(P_{2}+C_{2}\right)\right), \frac{1}{2}\left(L_{1}+\frac{1}{2}\left(P_{1}+C_{1}\right)\right)\right\rangle
$$

Ansatz:

$$
\left(x_{1}^{2}+x_{2}^{2}+x_{3}^{2}+u^{2}\right)^{1 / 2}=\varphi(\omega), \quad \omega=x_{0}
$$

Reduced equation:

$$
\left(\varphi^{\prime}-1\right)\left(\varphi^{\prime}+1\right) \varphi^{2}=0
$$

Solutions of the reduced equation:

$$
\varphi(\omega)=\varepsilon \omega+c_{1}, \varepsilon= \pm 1, \quad \varphi=0
$$

Solutions of the eikonal equation:

$$
\left(x_{1}^{2}+x_{2}^{2}+x_{3}^{2}+u^{2}\right)^{1 / 2}=\varepsilon x_{0}+c_{1}, \varepsilon= \pm 1, \quad x_{1}^{2}+x_{2}^{2}+x_{3}^{2}+u^{2}=0
$$




\subsubsection{Reductions to PDEs}

From the invariants of one nonconjugate subalgebra, we constructed the ansatz, which reduced the eikonal equation to PDEs.

$$
\left\langle-L_{3},-L_{2},-L_{1}\right\rangle
$$

Ansatz:

$$
u=\varphi\left(\omega_{1}, \omega_{2}\right), \quad \omega_{1}=x_{0}, \omega_{2}=\left(x_{1}^{2}+x_{2}^{2}+x_{3}^{2}\right)^{1 / 2}
$$

Reduced equation:

$$
\varphi_{1}^{2}-\varphi_{2}^{2}-1=0
$$

Solutions of the reduced equation:

$$
\varphi\left(\omega_{1}, \omega_{2}\right)=\varepsilon \sqrt{c_{2}^{2}+1} \omega_{1}+c_{2} \omega_{2}+c_{1}+c_{2}, \varepsilon= \pm 1
$$

Solutions of the eikonal equation:

$$
u=\varepsilon\left(c_{2}^{2}+1\right)^{1 / 2} x_{0}+c_{2}\left(x_{1}^{2}+x_{2}^{2}+x_{3}^{2}\right)^{1 / 2}+c_{1}+c_{2}, \varepsilon= \pm 1
$$

As we see, the reduced equation is PDEs. As above, the reason is that the corresponding subalgebra has rank two.

\section{Conclusions}

The relationship is studied between the structural properties of three-dimensional nonconjugate subalgebras of the Lie algebra of the group $P(1,4)$ and the types of the symmetry reductions of the eikonal equation to ODEs.

Below, we present some consequences resulting from that relationship.

- Reductions to algebraic equations are induced by some subalgebras of the types $3 A_{1}, A_{3,1}$;

- Reductions to linear ODEs are induced by some subalgebras of the types $3 A_{1}, A_{3,6}$;

- Reductions to equations, which can be split on two linear ODEs, are induced by some subalgebras of the types $3 A_{1}, A_{2} \oplus A_{1}, A_{3,1}, A_{3,3}, A_{3,6}, A_{3,7}^{a}, A_{3,9}$;

- Reductions to nonlinear ODEs are induced by subalgebras of the type $A_{3,2}$ as well as by some subalgebras of the types $3 A_{1}, A_{2} \oplus A_{1}, A_{3,1}, A_{3,3}, A_{3,6}, A_{3,7}^{a}$;

- Reductions to PDEs are induced by subalgebras of the type $A_{3,8}$ as well as by some subalgebras of the types $A_{3,6}, A_{3,9}$;

- From invariants of subalgebras of the type $A_{3,4}$ as well as of some subalgebras of the types $3 A_{1}$, $A_{2} \oplus A_{1}, A_{3,1}, A_{3,2}, A_{3,3}, A_{3,6}$, we cannot construct ansatzes reducing the eikonal equation;

- There are no nonconjugate subalgebras of the Lie algebra of the group $P(1,4)$ of the type $A_{3,5}$.

Author Contributions: The results obtained by Volodymyr Fedorchuk could be found in the subsections 3.1 and 3.2. The results obtained by Vasyl Fedorchuk were presented in the subsections 3.3-3.11.

Conflicts of Interest: The authors declare no conflict of interest.

\section{References}

1. Borovskikh, A.V. The eikonal equation in a nonhomogeneous medium. Dokl. Akad. Nauk SSSR 2003, 391, 587-590. (In Russian)

2. Borovskikh, A.V. Group classification of the eikonal equations for a three-dimensional nonhomogeneous medium. Mat. Sb. 2004, 195, 23-64 (In Russian); translation in Sb. Math. 2004, 195, 479-520. 
3. Borovskikh, A.V. Group classification of the eikonal equations for the wave equation in a nonhomogeneous medium. Sovrem. Mat. Prilozh. 2003, 10, 22-33 (In Russian); translation in J. Math. Sci. (N.Y.) 2005, 129, 3537-3549.

4. Borovskikh, A.V. The two-dimensional eikonal equation. Sibirsk. Mat. Zh. 2006, 47, 993-1018 (In Russian); translation in Siberian Math. J. 2006, 47, 813-834.

5. Popovich, R.O.; Egorchenko, I.A. Group classification of generalized eikonal equations. Ukrain. Mat. Zh. 2001, 53, 1513-1520 (In Ukrainian); translation in Ukrainian Math. J. 2001, 53, 1841-1850.

6. Borovskikh, A.V. Eikonal equations for an inhomogeneous anisotropic medium. Sovrem. Mat. Prilozh., Uravneniya Matematicheskoi Fiziki 2009, 64, 18-38 (In Russian); translation in J. Math. Sci. (N.Y.) 2010, 164, 859-880.

7. Rumer, Yu.B. Issledovaniya po 5-optike; Studies in 5-dimensional optics; Zap.-Sibir. Filial Akad. Nauk SSSR., Gosudarstv. Izdat. Tehn.-Teor. Lit.: Moscow, Russia, 1956; 152p. (In Russian)

8. Sheng, Q.; Guha, S.; Gonzalez, L.P. An exponential transformation based splitting method for fast computations of highly oscillatory solutions. J. Comput. Appl. Math. 2011, 235, 4452-4463.

9. Sheng, Q.; Guha, S.; Gonzalez, L.P. A short note on the asymptotic stability of an oscillation-free eikonal splitting method. Appl. Math. Lett. 2012, 25, 1539-1543.

10. Fushchich, W.I.; Shtelen, W.M.; Serov, N.I. Symmetry Analysis and Exact Solutions of Equations of Nonlinear Mathematical Physics; Kluver Academic Publishers: Dordrecht, The Netherlands, 1993; 435p.

11. Fushchich, W.I.; Shtelen, W.M. The symmetry and some exact solutions of the relativistic eikonal equation. Lett. Nuovo Cimento 1982, 34, 498-502.

12. Barannik, A.F.; Barannik, L.F.; Fushchich, V.I. Reduction and exact solutions of the eikonal equation. Ukrain. Mat. Zh. 1991, 43, 461-474 (In Russian); translation in Ukrainian Math. J. 1991, 43, 425-437.

13. Barannyk, L.F.; Fushchych, W.I. On Continuous Subgroups of the Conformal Group of the Minkowski space $R_{1, n}$; Inst. of Math. Acad. of Sci. of Ukrainian SSR: Kyiv, Ukraine, 1988; 48p, Preprint N 88-34. (In Russian)

14. Barannyk, A.F.; Barannyk, L.F.; Fushchych, W.I. Connected subgroups of the conformal group $C(1,4)$. Ukrain. Math. J. 1991, 43, 870-884.

15. Barannik, A.F. On reduction of the eikonal equation in the Minkowski space $R_{1,4}$. In Algebra-theoretic Analysis of Equations in Mathematical Physics; Akad. Nauk Ukrain. SSR, Inst. Mat.: Kiev, Ukraine, 1990; pp. 18-20. (In Russian)

16. Fushchich, V.I.; Fedorchuk, V.M.; Fedorchuk, I.M. Subgroup Structure of the Generalized Poincaré Group and Exact Solutions of Certain Nonlinear Wave Equations; Akad. Nauk Ukrain. SSR Inst. Mat.: Kiev, Ukraine, 1986; 36p, Preprint No. 27. (In Russian)

17. Fedorchuk, V.M.; Fedorchuk, I.M. Reduction and Exact Solutions of Five-dimensional Nonlinear Wave Equations; Akad. Nauk Ukrain. SSR Inst. Mat.: Kiev, Ukraine, 1988; 28p, Preprint No. 21. (In Russian)

18. Fedorchuk, I.M. Symmetry reduction and exact solutions of the eikonal equation. J. Nonlinear Math. Phys. 1996, 3, 474-477.

19. Fedorchuk, I.M. On new exact solutions of the eikonal equation. In Proceedings of the Fourth International Conference on Symmetry in Nonlinear Mathematical Physics, Kiev, Ukraine, 10-13 July 2001; pp. 136-139.

20. Fedorchuk, I.M. Reduction and some exact solutions of the eikonal equation. Symm. Nonlinear Math. Phys. 1997, V.1, 241-244.

21. Fedorchuk, V.M.; Fedorchuk, I.M.; Leibov, O.S. Reduction of the Born-Infeld, the Monge-Ampere and the eikonal equation to linear equations. Dokl. Akad. Nauk Ukrainy 1991, 11, $24-27$.

22. Fedorchuk, V. Symmetry Reduction and Exact Solutions of the Euler-Lagrange-Born-Infeld, Multidimensional Monge-Ampere and Eikonal Equations. J. Nonlinear Math. Phys. 1995, 2, 329-333.

23. Ovsiannikov, L.V. Group Analysis of Differential Equations; Academic Press: New York, NY, USA, 1982; $\mathrm{xvi}+416 \mathrm{p}$.

24. Olver, P.J. Applications of Lie Groups to Differential Equations; Springer-Verlag: New York, NY, USA, 1986; xxvi+497p.

25. Fedorchuk, V.M. Symmetry reduction and some exact solutions of a nonlinear five-dimensional wave equation. Ukr. Mat. Zh. 1996, 48, 573-576 (In Ukrainian); translation in Ukrainian Math. J. 1997, 48, 636-640.

26. Grundland, A.M.; Harnad, J.; Winternitz, P. Symmetry reduction for nonlinear relativistically invariant equations. J. Math. Phys. 1984, 25, 791-806. 
27. Nikitin, A.G.; Kuriksha, O. Group analysis of equations of axion electrodynamics. In Group Analysis of Differential Equations and Integrable Systems; Department of Mathematics and Statistics, University of Cyprus: Nicosia, Cyprus, 2011; pp. 152-163.

28. Nikitin, A.G.; Kuriksha, O. Invariant solutions for equations of axion electrodynamics. Commun. Nonlinear Sci. Numer. Simulat. 2012, 17, 4585-4601.

29. Fushchych, W.I.; Nikitin, A.G. Symmetries of Equations of Quantum Mechanics; Allerton Press Inc.: New York, NY, USA, 1994; xvi+465p.

30. Kadyshevskii, V.G. A new approach to the theory of electromagnetic interactions. Fiz. Element. Chastits Atom. Yadra 1980, 11, 5-39.

31. Fushchych, W.I.; Serov N.I. On some exact solutions of many-dimensional non-linear Euler-Lagrange equation. Dokl. Acad. Nauk SSSR 1984, 278, 847-851.

32. Fushchych, W.I.; Serov N.I. Symmetry and some exact solutions of many-dimensional Monge-Ampère equation. Dokl. Acad. Nauk SSSR 1983, 273, 543-546.

33. Patera, J.; Winternitz, P.; Zassenhaus, H. Continuous subgroups of the fundamental groups of physics. I. General method and the Poincaré group. J. Math. Phys. 1975, 16, 1597-1614.

34. Fedorchuk, V.M. Continuous Subgroups of the Inhomogeneous de Sitter Group P(1,4); Institute of Mathematics, Ukrainian Academy of Sciences: Kiev, Ukraine, 1978; Preprint No. 78.18. (In Russian)

35. Fedorchuk, V.M. Splitting subalgebras of the Lie algebra of the generalized Poincaré group $P(1,4)$. Ukr. Mat. Zh. 1979, 31, 717-722 (In Russian); translation in Ukr. Math. J. 1979, 31, 554-558.

36. Fedorchuk, V.M.; Fushchich, V.I. On subgroups of the generalized Poincare group. In Proceedings of the International Seminar on Group-Theoretic Methods in Physics, Zvenigorod, Russia, 28-30 November, 1979; pp. 61-66. (In Russian)

37. Fedorchuk, V.M. Nonsplit subalgebras of the Lie algebra of the generalized Poincaré group $P(1,4)$. Ukr. Mat. Zh. 1981, 33, 696-700 (In Russian); translation in Ukr. Math. J. 1981, 33, 535-538.

38. Fushchich, W.I.; Barannik, A.F.; Barannik, L.F.; Fedorchuk, V.M. Continuous subgroups of the Poincaré group P(1,4). J. Phys. A: Math. Gen. 1985, 18, 2893-2899.

39. Fushchich, W.I.; Nikitin, A.G. Reduction of the representations of the generalized Poincare algebra by the Galilei algebra. J. Phys. A: Math. Gen. 1980, 13, 2319-2330.

40. Fushchich, V.I.; Barannik, L.F.; Barannik, A.F. Subgroup Analysis of Galilei and Poincaré Groups and Reduction of Nonlinear Equations; Naukova Dumka: Kiev, Ukraine, 1991. (In Russian)

41. Mubarakzyanov, G.M. On solvable Lie algebras. Izv. Vyssh. Uchebn. Zaved., Ser. Mat. 1963, 1, 114-123.

42. Fedorchuk, V.M.; Fedorchuk, V.I. On classification of the low-dimensional non-conjugate subalgebras of the Lie algebra of the Poincaré group P(1,4). Proc. Inst. Math. NAS Ukr. 2006, 3, 302-308.

43. Patera, J.; Sharp, R.T.; Winternitz, P.; Zassenhaus, H. Invariants of real low dimension Lie algebras. J. Math. Phys. 1976, 17, 986-994.

44. Patera, J.; Sharp, R.T.; Winternitz, P.; Zassenhaus, H. Subgroups of the Poincaré group and their invariants. J. Math. Phys. 1976, 17, 977-985.

(C) 2016 by the authors; licensee MDPI, Basel, Switzerland. This article is an open access article distributed under the terms and conditions of the Creative Commons Attribution (CC-BY) license (http://creativecommons.org/licenses/by/4.0/). 